\title{
Analytical Analysis for Parameter Design of Attached Nonlinear Energy Sink
}

\author{
Tianjiao Zhang $(\mathbb{D}$, Luyu Li $\mathbb{D}$, and Yilin Zheng $(\mathbb{D}$ \\ School of Construction Engineering, Dalian University of Technology, Dalian, Liaoning 116024, China \\ Correspondence should be addressed to Tianjiao Zhang; 693550181@qq.com
}

Received 3 June 2021; Revised 5 July 2021; Accepted 17 July 2021; Published 3 August 2021

Academic Editor: Stefano Marchesiello

Copyright (c) 2021 Tianjiao Zhang et al. This is an open access article distributed under the Creative Commons Attribution License, which permits unrestricted use, distribution, and reproduction in any medium, provided the original work is properly cited.

\begin{abstract}
The dynamic responses of a linear primary structure coupled with a nonlinear energy sink (NES) are investigated under harmonic excitation in the $1: 1$ resonance regime. In civil engineering, initial conditions are usually zero or approximately zero. Therefore, in this study, only these conditions are considered. The strongly modulated response (SMR), whose occurrence is conditional, is the precondition for effective target energy transfer (TET) in this system. Therefore, this study aims to determine the parameter range in which the SMR can occur. The platform phenomenon and other related phenomena are observed while analyzing slow-varying equations. An excitation amplitude interval during which the SMR can occur is obtained, and an approximate analytical solution of the optimal nonlinear stiffness is found. The numerical results show that the NES based on the optimal stiffness performs better in terms of control performance.
\end{abstract}

\section{Introduction}

Natural disasters and man-made damages are considered in civil engineering. Hence, structural vibration control has become a research hotspot in recent years. Vibration control can be divided into active control, semiactive control, and passive control. The main differences between the three categories are the external energy requirement and the energy input method. Passive control devices have the simplest structure and lowest cost, as they do not require any external energy. Moreover, they have been widely investigated and applied for several years. The tuned mass damper (TMD) is the most representative type of passive control device that has been installed in several prominent buildings globally, e.g., the John Hancock Tower in Boston, Citicorp Center office building in New York, and Chiba Port Tower in Japan [1]. In China, a pendulum-type TMD coupled with a limiting stopper was installed in the Taipei Tower to control the vibration caused by winds and earthquakes [2].

In 1947, Den Hartog introduced damping in a dynamic vibration absorber and improved the control effect significantly. Den Hartog also provided optimization formulas for calculating the frequency and damping ratio of TMD [3].
Since then, the concept of TMD has been established. Tsai and Lin proposed an optimal parameter design method for TMDs based on Den Hartog's formulas and the fixed point theory [4]. Some scholars have proposed that in practical engineering, a TMD inherently exhibits nonlinear behavior owing to its large displacement or the application of a limiting stopper [5-7]. Li and Cui analyzed the nonlinearity in a TMD and proposed that the nonlinear behavior of the TMD should be considered during the design process to improve the control performance. They simplified the system to a single-degree-of-freedom system for analytical analysis and optimized the traditional design method [8]. Li and Zhang derived an optimal frequency formula for designing TMD and considered the nonlinear coefficient. They performed a numerical test on the optimized system and verified the superiority of the optimal method [9]. TMDs can function efficiently, depending on the tuning accuracy, and they absorb energy through the resonance of a primary structure. However, in practice, excitation frequencies are complex, and TMDs may lose their efficiency when the system deviates from the mode of resonance.

As linear dampers are very sensitive to tuning, more scholars are focusing on nonlinear dampers, which are also 
called nonlinear energy sinks (NESs). In 1952, Roberson pointed out that adding nonlinearity to a linear shock absorber can effectively increase the frequency bandwidth of vibration reduction [10]. The most widely used NESs are the particle dampers and nonlinear stiffness dampers. Particle dampers can be divided into four categories: one-unit singleparticle dampers [11], multiunit single particle dampers [12], one-unit multiparticle dampers [13], and multiunit multiparticle dampers [14]. Zheng et al. discussed in detail the development history, classification, structure, principle of particle dampers, and they also provided valuable references for future studies [15]. The nonlinear stiffness energy sink is another widely used damper. Gendelman et al. investigated the response regimes of a linear oscillator attached to an NES under external harmonic forcing. They used a phase diagram for global analysis and proposed a one-dimensional map that could be regarded as the sufficient and necessary condition for the strongly modulated response (SMR) [16-19]. Liu et al. studied the cubic stiffness nonlinear energy sink system under ground harmonic excitation, and the necessary condition and sufficient condition of strongly modulated response (SMR) are obtained [20]. Zhang et al. derived the relationship between the initial conditions and nonlinear stiffness for conservative systems using the Hamiltonian theory when the energy transfer was complete and extended the results to nonconservative systems to obtain the parametric condition for the optimal target energy transfer (TET) [21-26]. Shudeifat et al. proposed a negative stiffness NES that could be determined using a precompressed spring or inverted pendulum [27-29]. Manevitch et al. applied the Laplace transform and performed a numerical analysis to obtain an approximate analytical solution for the energy of the NES system [30]. Niu et al. studied the nonlinear dynamics of the simply supported FGM conical panel with different forms of initial imperfections [31]. Lu et al. explored the connection between the resonance response interaction and bubble-shaped response curve that may appear in the forced response of a nonlinear magnetoelectric coupled system and studied the resonance response interaction to enhance the vibratory energy harvesting bandwidth [32]. Tan et al. studied a system consisting of a ring isolator. The harmonic balance analysis was used to derive the frequency response functions of the transmissibility, and the effects of nonlinear stiffness, damping ratio, and mass were examined [33]. Qiu et al. proposed design criteria for the vibroimpact damper. The method was extended to the case of multiple VI NESs. The principles of additivity and separate activities of VI NESs are analyzed theoretically [34, 35].

NESs can resonate with the primary structure, but the mode will be destroyed instantly because nonlinear devices have no fixed frequency. Therefore, the energy transferred to NESs cannot return to the primary structure, and such a transfer pattern is considered as the TET. This transfer mode is considered as an advantage of nonlinear dampers. The occurrence of the SMR implies that a TET exists in the system, which guarantees NES to work effectively. However, the occurrence of SMR is conditional. The purpose of this study is to determine the range of NES parameters with which the system can cause an SMR.

Owing to the difficulty of nonlinear system analysis, most researchers have performed numerical analyses. Even when an approximate analytical solution of the optimal nonlinear stiffness can be obtained, the solution is based on the global analysis of the initial conditions, and the initial conditions are need to be known [21, 22]. However, the initial conditions are usually zero or approximately zero in civil engineering, and it cannot be measured in advance. Therefore, an innovative local optimization method is proposed in this study. The platform phenomenon caused by the modulation response is proposed and analyzed for the first time in this study. Based on this phenomenon, the range of modulation response of the nonlinear system is derived, through which the optimal stiffness of the NES independent of initial conditions can be obtained. The proposed method can avoid the problem that the derivation of the optimal nonlinear stiffness depends on the initial conditions.

A two-degree-of-freedom (2-DOF) system under harmonic excitation composed of a linear primary structure and cubic stiffness NES is investigated in this study. The first section is the introduction of the research work. In the second section, the motion equation and analytical analysis of the system are determined. A platform phenomenon and other related phenomena are found while establishing the variation laws of the amplitude calculated using the slowly varying equation with excitation in the third section. In the fourth section, based on the above phenomenon, the formulas for the excitation range in which SMRs can occur are derived when other parameters are fixed. In the fifth section, an optimal approximate analytical solution of the nonlinear stiffness is obtained, and the optimization effect is verified through numerical analysis. Finally, the last section is the conclusion of this work.

\section{Equations of Motion and Analytical Analysis}

The model used in this study is a 2-DOF system under harmonic excitation, which consisted of a primary linear oscillator and cubic stiffness NES, as shown in Figure 1. The subsequent investigations are all based on the initial conditions of approximately zero. The equations of motion are expressed as follows:

$$
\begin{aligned}
m_{1} \ddot{x}_{1}+c_{1} \dot{x}_{1}+k_{1} x_{1}+m_{2} \ddot{x}_{2} & =F \cos \omega t, \\
m_{2} \ddot{x}_{2}+c_{2}\left(\dot{x}_{2}-\dot{x}_{1}\right)+k_{2}\left(x_{2}-x_{1}\right)^{3} & =0,
\end{aligned}
$$

where $x_{1}$ and $x_{2}, c_{1}$ and $c_{2}$, and $k_{1}$ and $k_{2}$ are the absolute displacements, damping coefficients, and stiffness coefficients of the linear primary oscillator and NES, respectively. $F$ is the amplitude of excitation. The following dimensionless coefficients are introduced to simplify equations (1) and (2) further. 


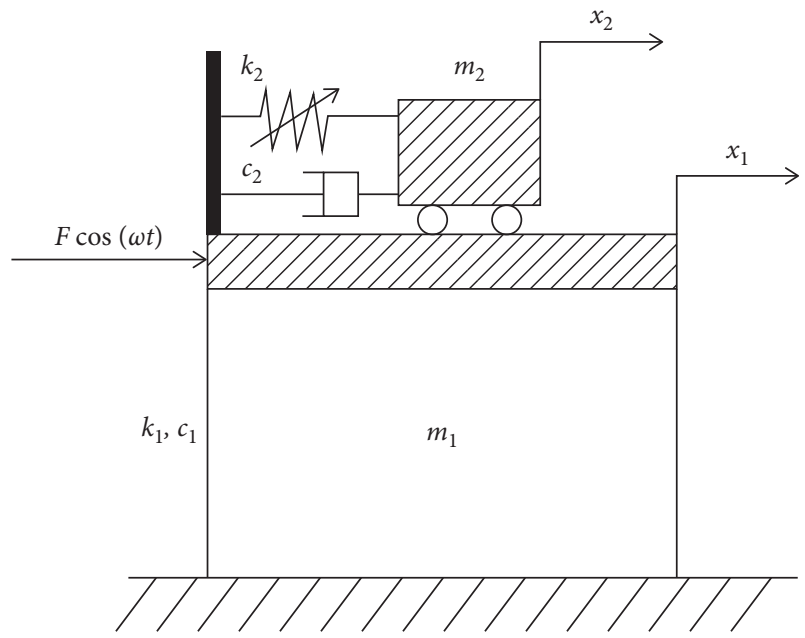

FIgURE 1: Simplified model of the 2-DOF system.

$$
\begin{aligned}
\frac{m_{2}}{m_{1}} & =\varepsilon, \\
\frac{c_{1}}{m_{1}} & =\varepsilon \lambda_{1}, \\
\frac{k_{1}}{m_{1}} & =\omega_{1}^{2}, \\
\frac{F}{m_{1}} & =\varepsilon f, \\
\frac{c_{2}}{m_{2}} & =\lambda_{2}, \\
\frac{k_{2}}{m_{2}} & =k_{n} .
\end{aligned}
$$

The above dimensionless parameters are substituted into equations (1) and (2), which transformed the motion equations into dimensionless forms, as shown in the following equations.

$$
\begin{aligned}
\ddot{x}_{1}+\varepsilon \lambda_{1} \dot{x}_{1}+\omega_{1}^{2} x_{1}+\varepsilon \ddot{x}_{2} & =\varepsilon f \cos \omega t, \\
\varepsilon\left[\ddot{x}_{2}+\lambda_{2}\left(\dot{x}_{2}-\dot{x}_{1}\right)+k_{n}\left(x_{2}-x_{1}\right)^{3}\right] & =0 .
\end{aligned}
$$

For the convenience of calculation, the absolute displacement coordinates of the primary structure and NES can be expressed using $u$ and $v$, as follows.

$$
\begin{aligned}
& u=x_{1}+\varepsilon x_{2}, \\
& v=x_{1}-x_{2} .
\end{aligned}
$$

The new coordinates are substituted into equations (4) and (5) to establish the motion equations.

$$
\begin{aligned}
& \ddot{u}+\frac{\varepsilon \lambda_{1}}{\varepsilon+1} \dot{u}+\frac{\varepsilon^{2} \lambda_{1}}{\varepsilon+1} \dot{v}+\frac{\omega_{1}^{2}}{1+\varepsilon} u+\frac{\varepsilon \omega_{1}^{2}}{1+\varepsilon} v=\varepsilon f \cos \omega t, \\
& \varepsilon\left[\ddot{u}-\ddot{v}-\lambda_{2}(\varepsilon+1) \dot{v}-(\varepsilon+1) k_{n} * v^{3}\right]=0 .
\end{aligned}
$$

For the convenience of calculation, the $\omega_{1}$ in the above formula is set as 1 , and the $0\left(\varepsilon^{2}\right)$ term in the system is eliminated. Then the equations (8) and (9) become

$$
\begin{gathered}
\ddot{u}+\frac{\varepsilon \lambda_{1}}{\varepsilon+1} \dot{u}+\frac{1}{\varepsilon+1}(u+\varepsilon v)=\varepsilon f \cos \omega t, \\
\varepsilon\left(\ddot{u}-\ddot{v}-\lambda_{2} \dot{v}-k n \cdot v^{3}\right)=0 .
\end{gathered}
$$

The differential equations (9) and (10) are simplified using Taylor's formula, such that each equation contains only one second-derivative term. By some algebraic derivations, equations (9) and (10) become

$$
\ddot{u}+u+\varepsilon\left(\lambda_{1} \dot{u}-u+v-f \cos \omega t\right)=0,
$$

$$
\varepsilon\left[\ddot{v}+\lambda_{2} \dot{v}+k n \cdot v^{3}+u+\varepsilon\left(\lambda_{1} \dot{u}-u+v-f \cos \omega t\right)\right]=0,
$$

omitting the higher-order terms $0\left(\varepsilon^{2}\right)$ in equation (12) gives

$$
\varepsilon\left(\ddot{v}+\lambda_{2} \dot{v}+k n \cdot v^{3}+u\right)=0
$$

The next step is a coordinate transformation procedure based on the complex variable average method (CX-A) to eliminate the fast variables because they have a minimal significance in our study. Therefore, the coordinates are converted into complex coordinates.

$$
\begin{aligned}
& \varphi_{1} e^{i \omega t}=\dot{u}+i \omega u, \\
& \varphi_{2} e^{i \omega t}=\dot{v}+i \omega v .
\end{aligned}
$$

The complex variables are substituted into equations (11) and (13). The original complex variable equations are as follows:

$$
\begin{array}{r}
\dot{\varphi}_{1} e^{i t}+\varepsilon\left[\frac{\lambda_{1}}{2}\left(\varphi_{1} e^{i t}+\varphi_{1}^{*} e^{-i t}\right)+\frac{i}{2}\left(\varphi_{1} e^{i t}-\varphi_{1}^{*} e^{-i t}\right)-\frac{i}{2}\left(\varphi_{2} e^{i t}-\varphi_{2}^{*} e^{-i t}\right)-\frac{f}{2}\left(e^{i \omega t}-e^{i \omega t}\right)\right]=0, \\
\varepsilon\left[\dot{\varphi}_{2} e^{i t}+\frac{i}{2}\left(\varphi_{2} e^{i t}-\varphi_{2}^{*} e^{-i t}\right)+\frac{\lambda_{2}}{2}\left(\varphi_{2} e^{i t}+\varphi_{2}^{*} e^{-i t}\right)+\frac{i}{8} k n\left(\varphi_{2} e^{i t}-\varphi_{2}^{*} e^{-i t}\right)^{3}-\frac{i}{2}\left(\varphi_{1} e^{i t}-\varphi_{1}^{*} e^{-i t}\right)\right]=0 .
\end{array}
$$


Considering the tuning parameter between the primary structure and the excitation as $\sigma$, the frequency $\omega$ can be written as

$$
\omega=1+\varepsilon \sigma .
$$

After performing the average process and omitting higher-order terms, the following equations are obtained.

$$
\begin{aligned}
\dot{\varphi}_{1}+\frac{\varepsilon}{2}\left(\lambda_{1} \varphi_{1}+i \varphi_{1}-i \varphi_{2}-f e^{i \varepsilon \sigma t}\right) & =0, \\
\frac{\varepsilon}{2}\left(2 \dot{\varphi}_{2}+\lambda_{2} \varphi_{2}+i \varphi_{2}-i \varphi_{1}-\frac{3}{4} k n \cdot i\left|\varphi_{2}\right|^{2} \varphi_{2}\right) & =0 .
\end{aligned}
$$

Next, new complex variables are defined $\left(\psi_{1}=\varphi_{1}\right.$ $\left.e^{-i \varepsilon \sigma t}, \psi_{2}=\varphi_{2} e^{-i \varepsilon \sigma t}\right)$ and substituted into the above equations.

$$
\dot{\psi}_{1}+i \varepsilon \sigma \psi_{1}+\frac{\varepsilon}{2}\left(\lambda_{1} \psi_{1}+i \psi_{1}-i \psi_{2}-f\right)=0
$$

$$
\frac{\varepsilon}{2}\left(2 \dot{\psi}_{2}+2 i \varepsilon \sigma \psi_{2}+\lambda_{2} \psi_{2}+i \psi_{2}-i \psi_{1}-\frac{3}{4} k n \cdot i\left|\psi_{2}\right|^{2} \psi_{2}\right)=0
$$

The time-history curves are obtained using exact equations (7) and (8) and complex variable equations (18) and (19) to verify the accuracy of the complex variable motions.

It can be observed from Figures 2 and 3 that the amplitudes calculated using the original equations and the complex variable equations are almost consistent, although the phase deviates slightly. However, this study focuses mainly on the amplitude. Therefore, the complex variable equations (18) and (19) are sufficiently precise to be investigated further. The response form shown in Figures 2 and 3 is called SMR, which indicates that the steady-state response is unstable, the amplitude of the steady-state response is not constant, and the modulation intensity is deep (it is called weakly modulated resonant (WMR) when the modulation intensity is weak). The appearance of the SMR indicates that effective TET occurs between the primary structure and NES. Therefore, the SMR is the response mode that should be achieved in this study. Subsequent investigations are aimed at adjusting the parameters to obtain an SMR.

Based on a multiscale method, the equation can be divided into two dimensions as fast and slow. The fast and slow dimensions are relative concepts that can be subdivided infinitely in theory. The time variable is divided into two scales according to the accuracy requirements of this study.

$$
t=\tau_{0}+\varepsilon \tau_{1} .
$$

Thus, the derivative can be written as follows.

$$
D=D_{0}+\varepsilon D_{1} \text {. }
$$

By substituting equation (21) into equations (18) and (19), the following equations are obtained.

$$
D_{0} \psi_{1}+\varepsilon D_{1} \psi_{1}+i \varepsilon \sigma \psi_{1}+\frac{\varepsilon}{2}\left(\lambda_{1} \psi_{1}+i \psi_{1}-i \psi_{2}-f\right)=0,
$$

$$
\begin{aligned}
& \frac{\varepsilon}{2}\left[2\left(D_{0} \psi_{2}+\varepsilon D_{1} \psi_{2}\right)+2 i \varepsilon \sigma \psi_{2}+\lambda_{2} \psi_{2}+i \psi_{2}-i \psi_{1}\right. \\
& \left.\quad-\frac{3}{4} i k n\left|\psi_{2}\right|^{2} \psi_{2}\right]=0
\end{aligned}
$$

Equations (22) and (23) are considered as polynomials of $\varepsilon$, and the coefficients of the first and second powers of $\varepsilon$ are deduced as follows.

$$
\begin{aligned}
\varepsilon^{0}: D_{0} \psi_{1} & =0, \\
\varepsilon^{1}: D_{1} \psi_{1}+i \sigma \psi_{1}+\frac{1}{2}\left(\lambda_{1} \psi_{1}+i \psi_{1}-i \psi_{2}-f\right) & =0, \\
\frac{1}{2}\left(2 D_{0} \psi_{2}+\lambda_{2} \psi_{2}+i \psi_{2}-i \psi_{1}-\frac{3}{4} i k n\left|\psi_{2}\right|^{2} \psi_{2}\right) & =0 .
\end{aligned}
$$

As the slow variables of the steady-state response are analyzed mainly, the derivatives of the large time scale $\tau_{0}$ are ignored.

$$
\psi_{1}=\psi_{2}\left(1-i \lambda_{2}-\frac{3}{4} k n\left|\psi_{2}\right|^{2}\right)
$$

The complex variables are written in the form of polar coordinates, where the slowly varying amplitude and phase of NES are represented, as follows.

$$
\begin{aligned}
& \psi_{1}=N_{1} e^{i \delta_{1}}, \\
& \psi_{2}=N_{2} e^{i \delta_{2}}, \\
& Z_{1}=N_{1}^{2}, \\
& Z_{2}=N_{2}^{2} .
\end{aligned}
$$

By substituting from equations (28)-(31) into (27) and separating the real and imaginary parts, the following equation can be obtained.

$$
Z_{1}=Z_{2}\left[\left(\lambda_{2}\right)^{2}+\left(1-\frac{3 k n Z_{2}}{4}\right)^{2}\right]
$$

Therefore, the slow invariant manifold (SIM) can be obtained by (32) as the thick line in Figure 4, and the thin line is the actual response of complex variable equations (18) and (19). It can be observed that the steady-state part of the system always operated according to the SIM. However, when the response reaches the jump point, the system jumps at the jump points, and the steady-state response is no longer stable, jumping between the two steady-state branches is inconsistent with the SIM. It can be inferred that when the fast-scale derivative is set to zero, and only the results of the stable steady-state response is accurate. However, jumping is a rapidly varying behavior whose response path may not be adequately simulated. This 


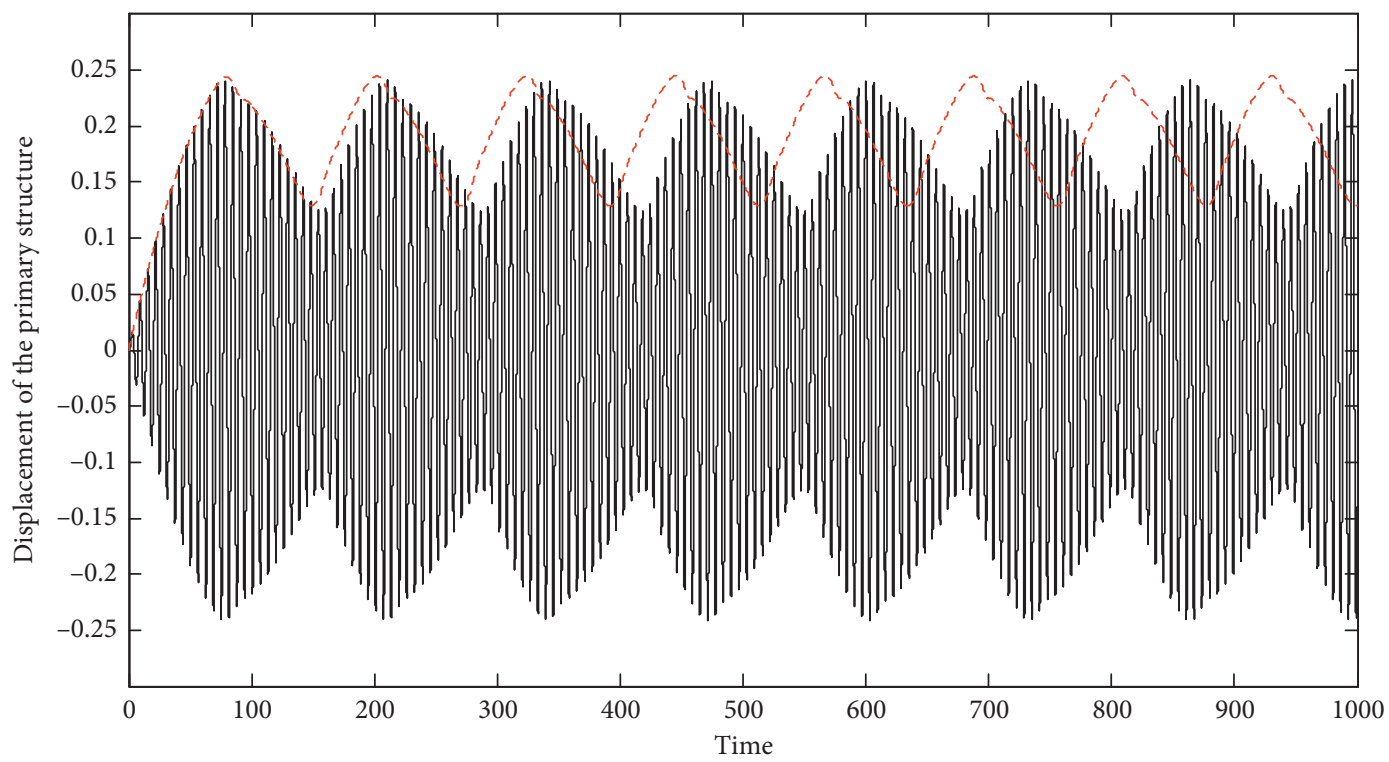

- Exact equation

- - Complexification averaging method

FIgURE 2: Time-history curves of the primary structure for the case $\lambda_{1}=0.8 ; \lambda_{2}=0.3 ; k n=5 ; f=0.5$.

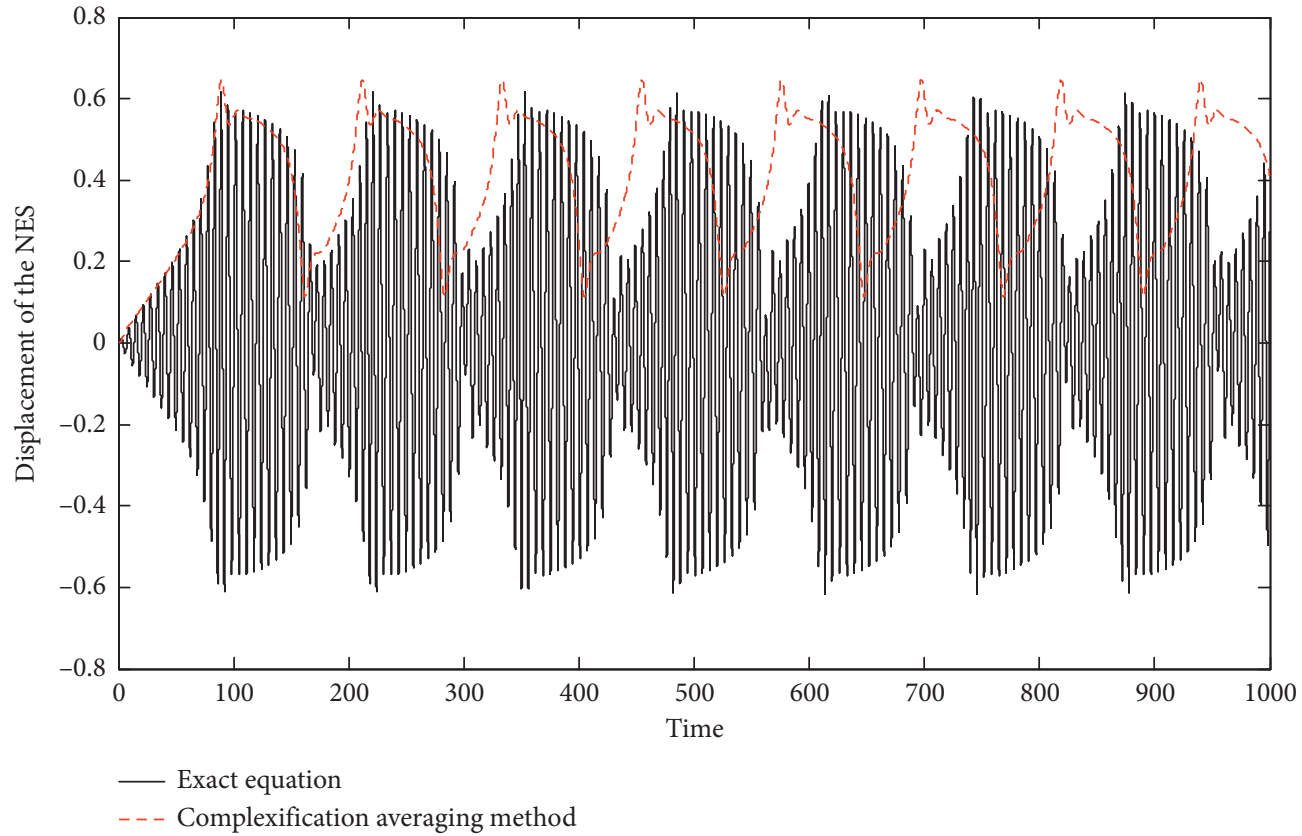

FIGURE 3: Time-history curves of the nonlinear energy sink $\lambda_{1}=0.8 ; \lambda_{2}=0.3 ; k n=5 ; f=0.5$.

deduction, which is also the theoretical basis of the following equation derivation, is confirmed later while deriving the slow-varying equations.

Considering the derivative of the above equation with respect to $Z_{2}$ to determine the jump points on the SIM and set this derivative as zero to obtain the following expression.

$$
\lambda_{2}^{2}+\left(1-\frac{3 k_{n} Z_{2}}{4}\right)\left(1-\frac{9 k n}{4} Z_{2}\right)=0
$$

Hence, the two local extremum points (jump points) can be solved as follows:

$$
\begin{aligned}
& N_{21}=\frac{2}{3 \sqrt{k n}} \sqrt{2+\sqrt{1-3 \lambda_{2}^{2}}}, \\
& N_{22}=\frac{2}{3 \sqrt{k n}} \sqrt{2-\sqrt{1-3 \lambda_{2}^{2}}} .
\end{aligned}
$$




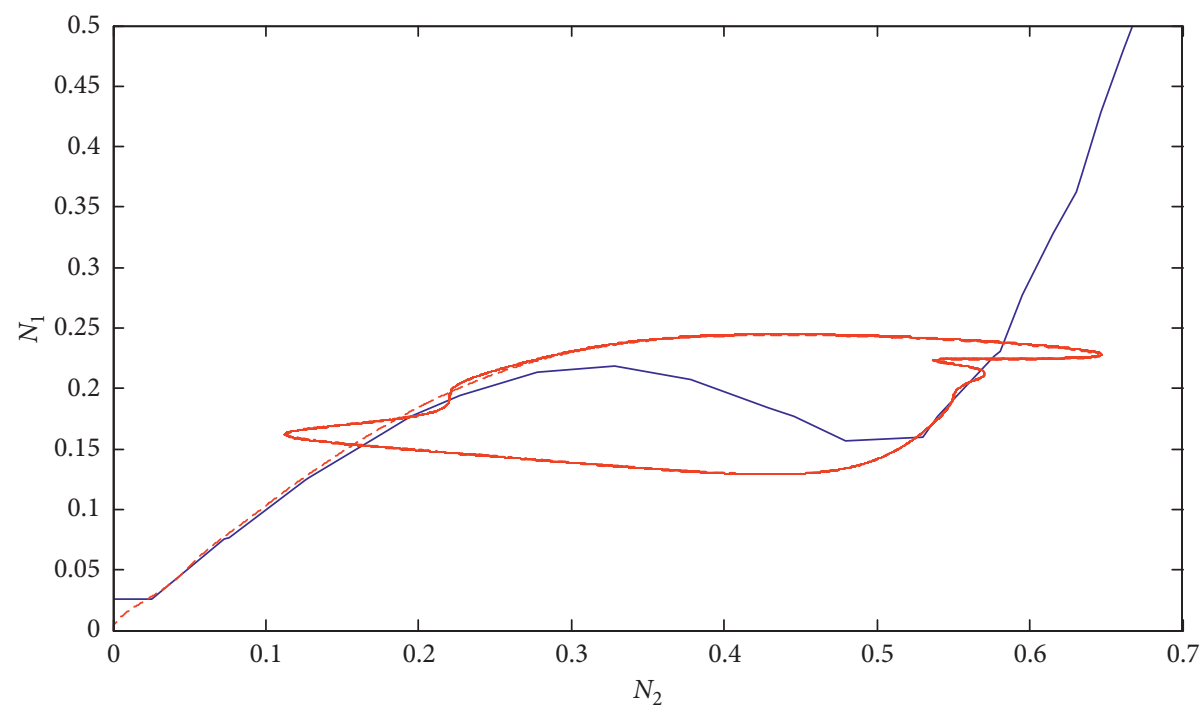

- Slow invariant manifold
--- Complex equation

Figure 4: Slow invariant manifold for the case $\lambda_{1}=0.8 ; \lambda_{2}=0.3 ; k n=5 ; f=0.5$.

It can be observed from equations (34) and (35) that the two extreme points can appear if only the damping condition satisfies $\lambda_{2} \leq \sqrt{3} / 3$. If $\lambda_{2}$ does not satisfy this inequality, then the jump phenomena will not occur in the system regardless of how the other parameters change. On the basis that damping satisfies the above conditions, the SMR occurrence depends on the value of nonlinear stiffness $k n$. However, critical stiffness is related to excitation amplitude.
If the jump occurs in the system, $N_{21}, N_{22}$ are the two jumping points, and they are known as the saddle junction bifurcation (SN bifurcation). In the actual response, where the jump occurs depends on the initial conditions. The initial conditions are usually approximately zero in civil engineering, and it can be inferred from the SIM that the jumping point should be $N_{21}$ in this case. Therefore, $N_{21}$ was directly considered as the $\mathrm{SN}$ bifurcation point later.

Substituting equation (27) into equation (25), a slowly varying equation for $\psi_{2}$ is obtained as follows:

$$
\left(1-\lambda_{2} i-\frac{3}{2} k n\left|\psi_{2}\right|^{2}\right) \frac{\partial \psi_{2}}{\partial \tau_{1}}-\frac{3}{4} k n \psi_{2}^{2} \frac{\partial \psi_{2}^{*}}{\partial \tau_{1}}+\frac{\psi_{2}}{2}\left[\lambda_{1}+2 i \sigma-\left(\lambda_{1}+2 i \sigma+i\right)\left(\lambda_{2} i+\frac{3}{4} k n\left|\psi_{2}\right|^{2}\right)\right]=\frac{f}{2}
$$

By solving the above equations, the following equation is obtained:

$$
\frac{\partial \psi_{2}}{\partial \tau_{1}}=\frac{A^{*} G-B G^{*}}{|A|^{2}-|B|^{2}}
$$

Among them,

$$
\begin{aligned}
& A=1-\lambda_{2} i-\frac{3}{2} k n\left|\psi_{2}\right|^{2}, \\
& B=-\frac{3}{4} k n \psi_{2}^{2}, \\
& G=\frac{f}{2}-\frac{\psi_{2}}{2}\left[\lambda_{1}+2 i \sigma-\left(\lambda_{1}+2 i \sigma+i\right)\left(\lambda_{2} i+\frac{3}{4} k n\left|\psi_{2}\right|^{2}\right)\right] .
\end{aligned}
$$

The variable $\psi_{2}$ was written as an exponential form $\mathrm{N}_{2} e^{i \delta_{2}}$ and substituted into the above equations. By separating the real and imaginary parts, the following equations can be obtained.

$$
\begin{aligned}
& \frac{\partial N_{2}}{\partial \tau_{1}}=\frac{1}{2 M}\left[\left(1-\frac{3}{4} k n N_{2}^{2}\right) C+\lambda_{2} D\right], \\
& \frac{\partial \delta_{2}}{\partial \tau_{1}}=\frac{1}{2 M N_{2}}\left[\lambda_{2} C-\left(1-\frac{9}{4} k n N_{2}^{2}\right) D\right] .
\end{aligned}
$$

Among them,

$$
\begin{aligned}
& C=f \cos \delta_{2}-N_{2}\left(\lambda_{1}+\lambda_{2}+2 \sigma \lambda_{2}-\frac{3}{4} k n \lambda_{1} N_{2}^{2}\right) \\
& D=f \sin \delta_{2}+N_{2}\left(2 \sigma-\lambda_{1} \lambda_{2}-\frac{3}{2} \sigma k n N_{2}^{2}-\frac{3}{4} k n N_{2}^{2}\right) \\
& M=\frac{27}{16} k n^{2} N_{2}^{4}-3 k n N_{2}^{2}+\lambda_{2}^{2}+1 .
\end{aligned}
$$


Equations (39) and (40) can be expressed as follows:

$$
\begin{gathered}
\frac{\partial N_{2}}{\partial \tau_{1}}=\frac{h_{1}}{M}, \\
\frac{\partial \delta_{2}}{\partial \tau_{1}}=\frac{h_{2}}{M} .
\end{gathered}
$$

Among them,

$$
\begin{aligned}
& h_{1}=\frac{1}{2}\left[\left(1-\frac{3}{4} k n N_{2}^{2}\right) C+\lambda_{2} D\right], \\
& h_{2}=\frac{1}{2 N_{2}}\left[\lambda_{2} C-\left(1-\frac{9}{4} k n N_{2}^{2}\right) D\right] .
\end{aligned}
$$

The denominator $M$ will result in a singularity at the jumping points; hence, the system cannot be simulated normally. In order to overcome this problem, rescaling the time by the term $M$ [9] yields the following equations.

$$
\begin{aligned}
N_{2}^{\prime} & =f_{1}, \\
\delta_{2}^{\prime} & =f_{2},
\end{aligned}
$$

where ${ }^{\prime}$ is the derivative with respect to the rescaled time.

\section{Platform Phenomenon and Effective Range of Excitation Amplitude}

The accuracy of equations (43) and (44) is verified by comparing their solutions with the result of the original equation. Consider the SIMs, time-history curves, and phase diagrams of the system. In Figures 5 and 6, the solid bold lines and dashed bold lines are obtained using complex variable equations (18) and (19) and slow-varying equations (43) and (44), respectively. Since it is not easy to adjust the time scale for the slow-varying equations, only the steadystate response of the slow-varying equations is plotted for comparison purpose. The solid thin lines in Figures 5 and 6 are obtained using the slow invariant manifold equation (32). The excitation amplitude of Figures 5-7 is 0.25 ; at this moment, the system amplitude do not reach the jump point yet; hence, no jump occurred. The steady-state response of the system remained stable in the region of the low amplitude, as shown in Figure 5.

The excitation amplitude in Figures $8-10$ is $f=1$. In Figures 8 and 9, the solid bold lines and dashed bold lines are obtained using complex variable equations (18) and (19) and slow-varying equations (43) and (44), respectively. The solid thin line shown in Figures 8 and 9 is obtained using the slow invariant manifold equation (32). Here, the system amplitude has experienced the jump point. Only one jump appears, and then, the steady-state response of the system, which always stays within the region of the high amplitude (Figure 8), becomes stable again.

As the jump corresponded to the fast behavior, equations (43) and (44), in which rapid-varying variables are ignored, cannot describe the jump accurately. As shown in Figures 11 and 12, the solid bold lines and dashed bold lines are obtained using complex variable equations (18) and (19) and slow-varying equations (43) and (44), respectively. The solid thin lines in Figures 11 and 12 are obtained using the slow invariant manifold equation (32). The amplitude $\mathrm{N}_{2}$ obtains using equations (43) and (44), experiences a rapid increase, and finally stays at point $N_{21}$ (the jump point). The excitation amplitude of Figures 11-13 is 0.5.

In this case, it appears impossible to use the slow-varying equations (43) and (44) to analyze the SMR. However, the parameter region of SMR can be solved by using the limitation of slowly varying equations. In the three sets of figures, the stiffness $k n$ is constant, but the excitation amplitude $f$ is different. The steady-state response is stable when $f=0.25 ; f=0.8$, and the steady-state response is the SMR when $f=0.5$. The steady-state response is stable again when $f=1$. Therefore, it is speculated that when the nonlinear stiffness $k n$ is fixed, an interval of excitation amplitude exists, and the SMR occurs only in this interval. The upper and lower limits of the interval could be related to $k n$. The nonlinear stiffness is considered as $k n=3,5,8,11$ to verify this inference. The steady-state amplitude $N_{2}$ is calculated using equations (43) and (44), as shown in Figure 14.

Figure 14 shows the above conjecture regarding a particular interval in which the SMR can occur. Moreover, in Figure 14, a unique platform region can be observed, and the value of the vertical axis corresponding to the platform is precisely the SN bifurcation point $N_{21}$. The SMR occurred when $f$ is in this platform. Furthermore, when the platform scope is exceeded, the steady-state response of the system is stable again. The jump behavior will not occur in the monotonically increasing region in front of the platform, and the amplitude of the steady-state response remains in the low-amplitude region. The monotonically increasing region behind the platform is attracted by a stable attractor in the high-amplitude region after a few jumps, and the steady-state response is also stable. However, neither of these cases has sustained SMRs. Therefore, they are regarded as invalid.

Consider parameters $\lambda_{1}=0.8, \lambda_{2}=0.3, k n=5$, along with $f$ in front of the platform and behind the platform, i.e., $f=0.2, f=0.4, f=0.6, f=0.8$, and verify the above phenomenon in Figures 15-18. In Figures 15-18, the solid thin lines are obtained using equations (7) and (8), solid bold lines using complex variable equations (18) and (19), and dashed bold lines using slow-varying equations (43) and (44).

The cause of the platform is similar to that mentioned above. When the excitation amplitude is small, the steadystate amplitude do not reach the jump point. Therefore, the system presents a stable steady-state response in the region of the low amplitude. When the SMR occurs, equations (43) and (44), in which the fast-varying parts are ignored, cannot be used to simulate the jumping process accurately. After experiencing a rapid increase within the first few seconds, the response finally stays at the jump point. The value of the jump point determined using equation (34) is related to $k n$ and $\lambda_{2}$ and independent of $f$. Therefore, in the SMR process, the rate of change of the amplitude $N_{2}$ with the excitation amplitude is zero. When the amplitude of excitation continues to increase, the system will jump to the 


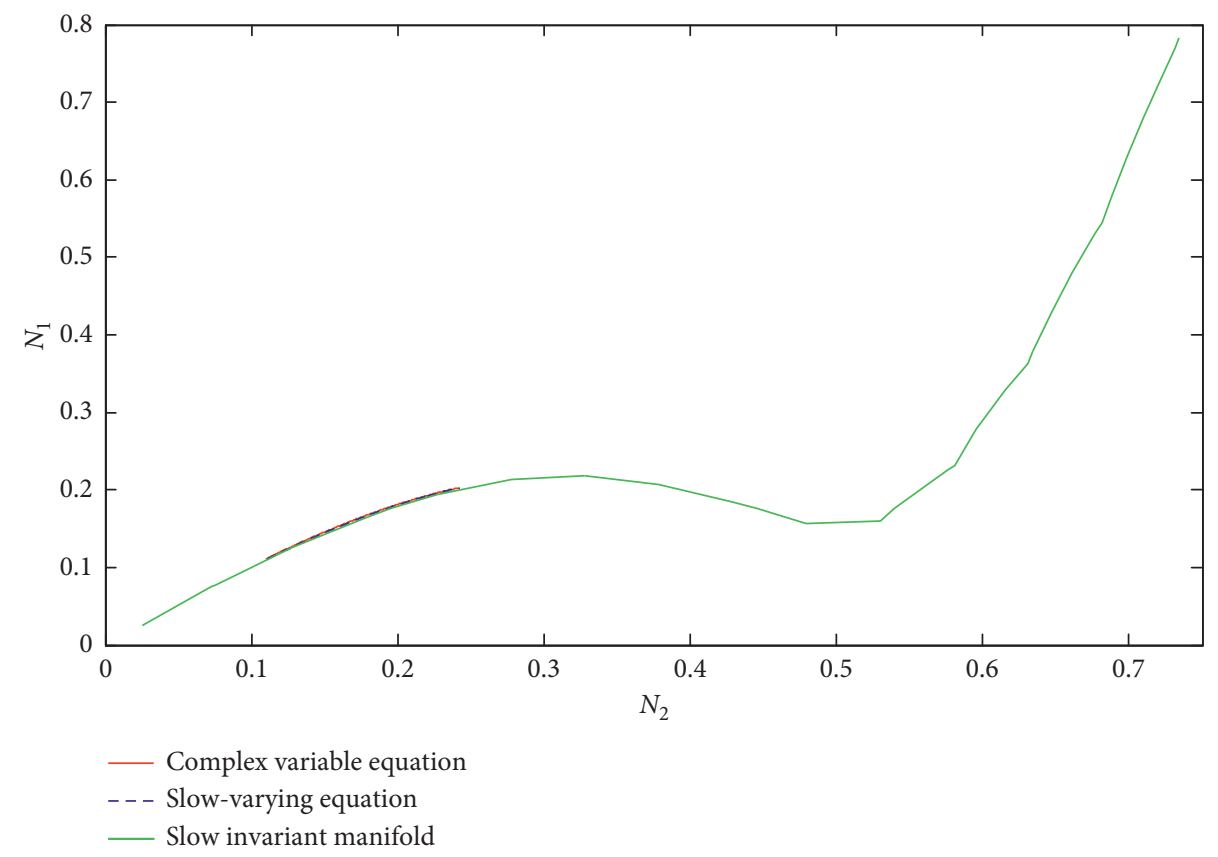

Figure 5: Slow invariant manifold for the case $\lambda_{1}=0.8 ; \lambda_{2}=0.3 ; k n=5 ; f=0.25$.
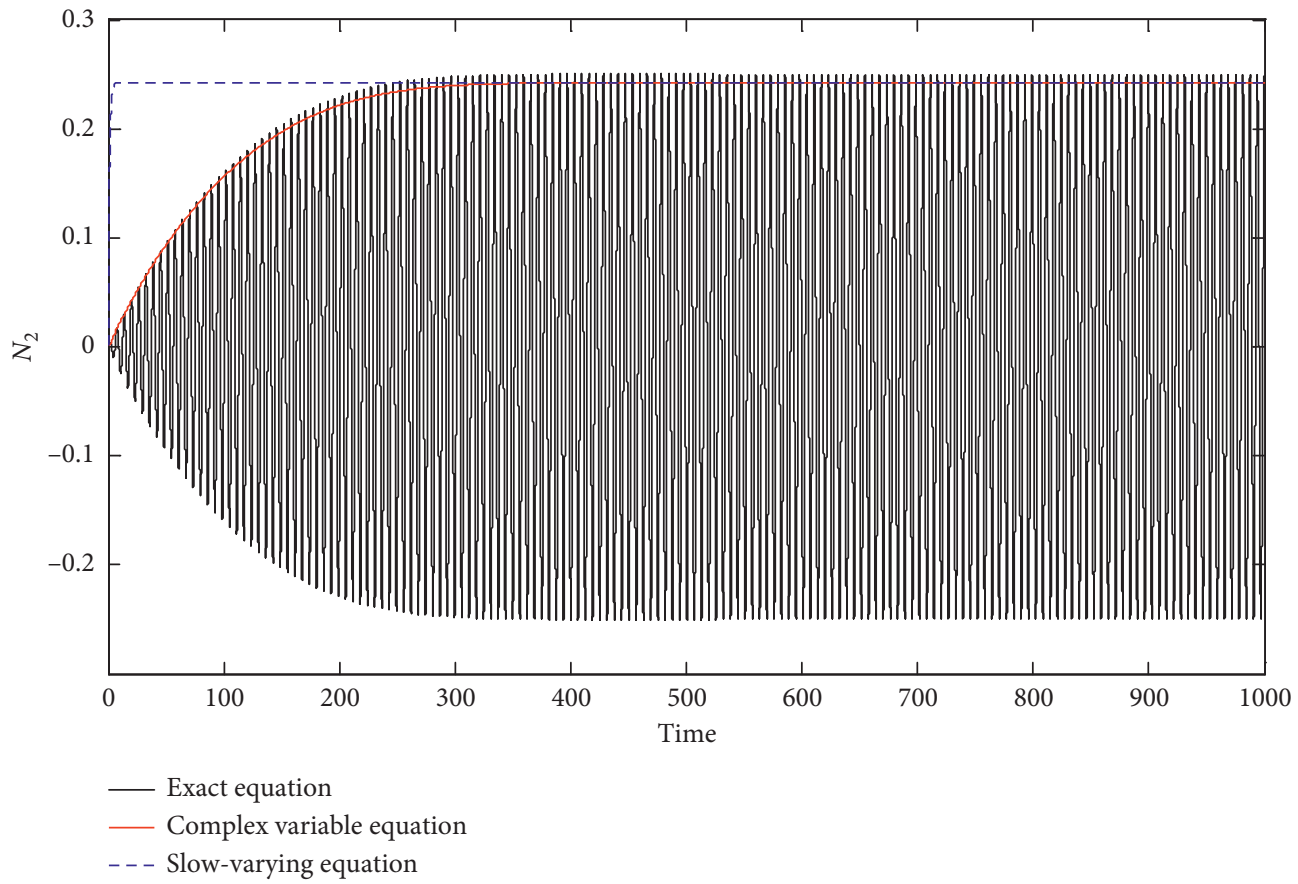

FIGURE 6: Time-history curves of nonlinear energy sink. The dashed line refers to the steady-state response of the slow-varying equation. System parameters: $\lambda_{1}=0.8 ; \lambda_{2}=0.3 ; k n=5 ; f=0.25$.

high-amplitude branch on the SIM. However, no SMR occurs. Therefore, the stable steady-state response will appear, and the slowly varying equation is able to simulate the amplitude accurately again. Moreover, when the other parameters are fixed, the $x$-coordinates of the starting and ending points of the platform corresponded to the upper and lower limits of the SMR excitation amplitude, respectively. This interval is set as $\left[f_{1}, f_{2}\right]$, within which an effective TET occurs. It can be observed from Figure 14 that the value of $\left[f_{1}, f_{2}\right]$ is related to $k n$.

The change in the phase $\delta_{2}$ with $f$ is shown in Figure 19. It is found that $\delta_{2}$ decreases monotonically only in the platform regions. Another phenomenon that can be observed is that the ordinates corresponding to the monotonically decreasing interval are independent of $k n$, and the phases at the starting and ending points of the 


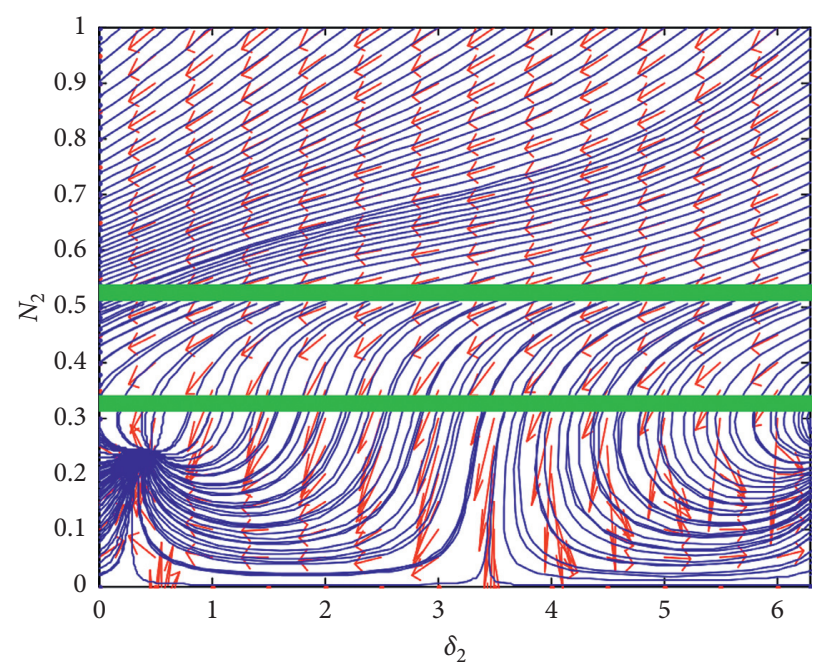

FIgURE 7: Phase portrait of nonlinear energy sink for the case $\lambda_{1}=0.8 ; \lambda_{2}=0.3 ; k n=5 ; f=0.25$.

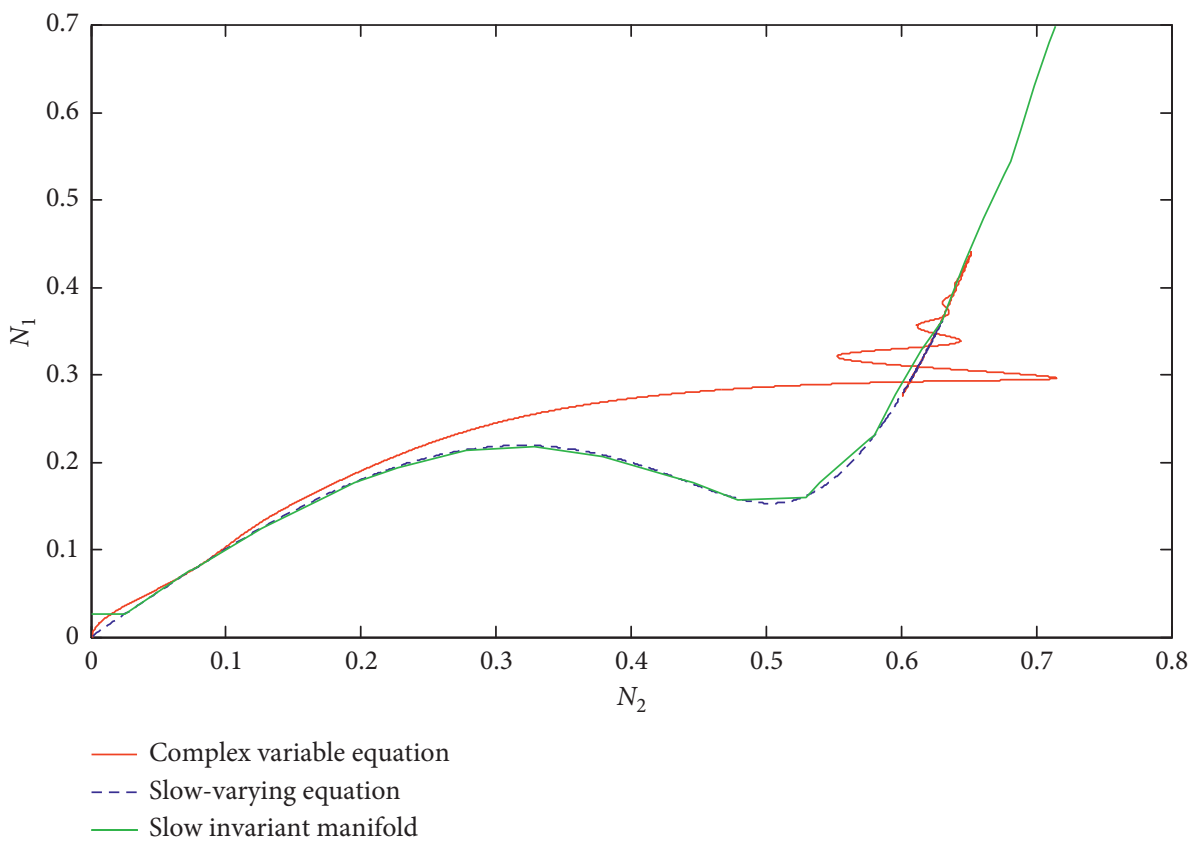

FIgURE 8: Slow invariant manifold for the case $\lambda_{1}=0.8 ; \lambda_{2}=0.3 ; k n=5 ; f=1$.

monotonically decreasing region can be adopted as negative.

By analyzing the time-history curves obtained using the slow-varying equations (43) and (44), it can be observed that $N_{2}^{\prime}=\delta_{2}^{\prime}=0$ is always precise during the steady-state response. Therefore, it can be inferred that the numerators of $h_{1}$ and $h_{2}$ are always zero in this case.

$$
\begin{aligned}
& \left(1-\frac{3}{4} k n N_{2}^{2}\right) C+\lambda_{2} D=0, \\
& \lambda_{2} C-\left(1-\frac{9}{4} k n N_{2}^{2}\right) D=0 .
\end{aligned}
$$

For convenience, equations (39) and (40) can be rewritten as follows.

$$
\begin{aligned}
& C=f \cos \delta_{2}-N_{2}\left(\lambda_{1}+\lambda_{2}+2 \sigma \lambda_{2}-\frac{3}{4} k n \lambda_{1} N_{2}^{2}\right), \\
& D=f \sin \delta_{2}+N_{2}\left(2 \sigma-\lambda_{1} \lambda_{2}-\frac{3}{2} \sigma k n N_{2}^{2}-\frac{3}{4} k n N_{2}^{2}\right) .
\end{aligned}
$$

As $N_{2}^{\prime}=\delta_{2}^{\prime}=0$, it can be deduced that $C=D=0$. This assumption is realistic when the steady-state response is stable. However, when the parameters are in the platform region, as shown in Figure 14, it becomes impossible to obtain $C=D=0 . N_{2}$ calculated from equations (43) and (44) is fixed, and the value of $\cos \delta_{2}$ increases monotonically with the change in $f$. Therefore, it is impossible that $C$ and $D$ are unchanged when $f$ increased. The time-history curves in 


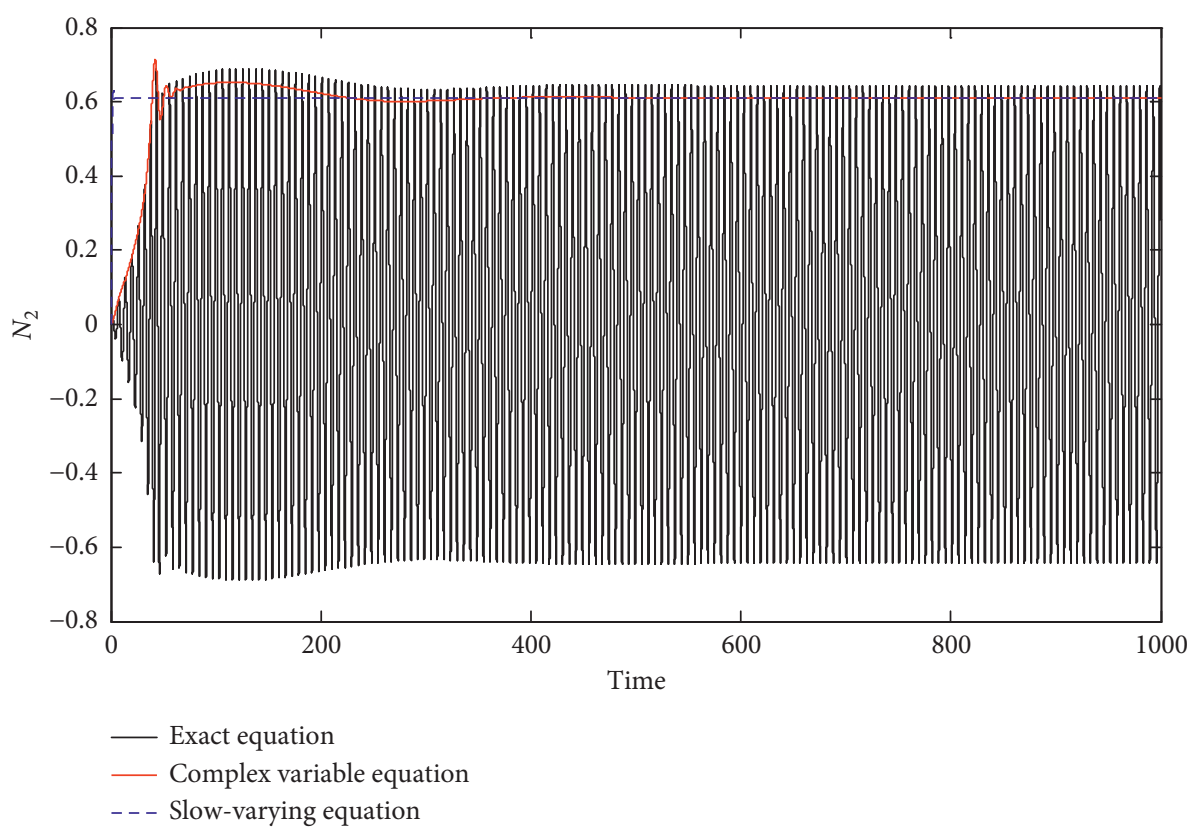

Figure 9: Time-history curves of nonlinear energy sink. The dashed line refers to the steady-state response of the slow-varying equation. System parameters: $\lambda_{1}=0.8 ; \lambda_{2}=0.3 ; k n=5 ; f=1$.

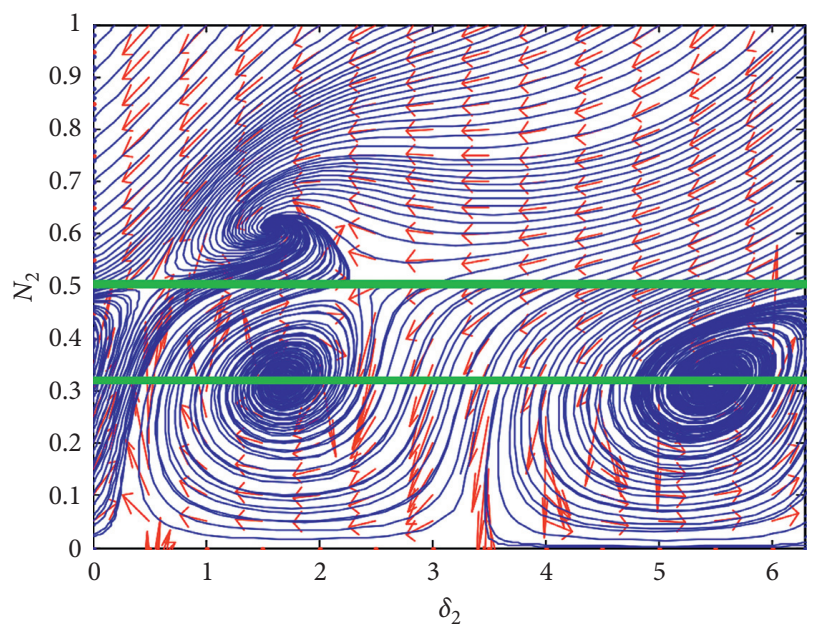

Figure 10: Phase portrait of nonlinear energy sink for the case $\lambda_{1}=0.8 ; \lambda_{2}=0.3 ; k n=5 ; f=1$.

Figure 20 show that when the steady-state response is stable, and the values of $C$ and $D$ are always zero. However, $C$ and $D$ are not zero only in the platform where the SMR occurs. Moreover, the $x$-coordinate intervals of $C$ and $D$ are not zero. The $x$-coordinate interval in which $\delta_{2}$ decreases monotonically and the $x$-coordinate interval of the platform coincide, it is all $\left[f_{1}, f_{2}\right]$. The above expressions and conditions could be used to obtain an approximate analytical solution for the range of excitation amplitude for the SMR. The following expressions are assumed:

$$
\begin{aligned}
& F_{1}=\left(\lambda_{1}+\lambda_{2}+2 \sigma \lambda_{2}-\frac{3}{4} k n \lambda_{1} N_{21}^{2}\right) N_{21}, \\
& F_{2}=\left(2 \sigma-\lambda_{1} \lambda_{2}-\frac{3}{2} \sigma k n N_{21}^{2}-\frac{3}{4} k n N_{21}^{2}\right) N_{21} .
\end{aligned}
$$

At the starting point of the platform, $C, D$ is still equal to zero. Therefore, equation (50) can be derived from equations (46) and (47).

$$
f_{1}^{2}=F_{1}^{2}+F_{2}^{2}
$$

From equation (50), the lower limit $f_{1}$ of the platform is obtained. The upper limit $f_{2}$ is calculated by setting the values of $C$ and $D$ at the starting and ending points as $C_{1}, C_{2}, D_{1}, D_{2}$, respectively.

$$
\begin{aligned}
& C_{1}=f_{1} \cos \delta_{21}-F_{1}=0, \\
& D_{1}=f_{1} \sin \delta_{21}+F_{2}=0, \\
& C_{2}=f_{2} \cos \delta_{21}-F_{1}, \\
& D_{2}=-f_{2} \sin \delta_{21}+F_{2} .
\end{aligned}
$$
(51).

The starting point phase can be obtained from equation

$$
\delta_{21}=\arccos \frac{F_{1}}{f_{1}} .
$$

From equations (51)-(54), the following expressions are derived. 


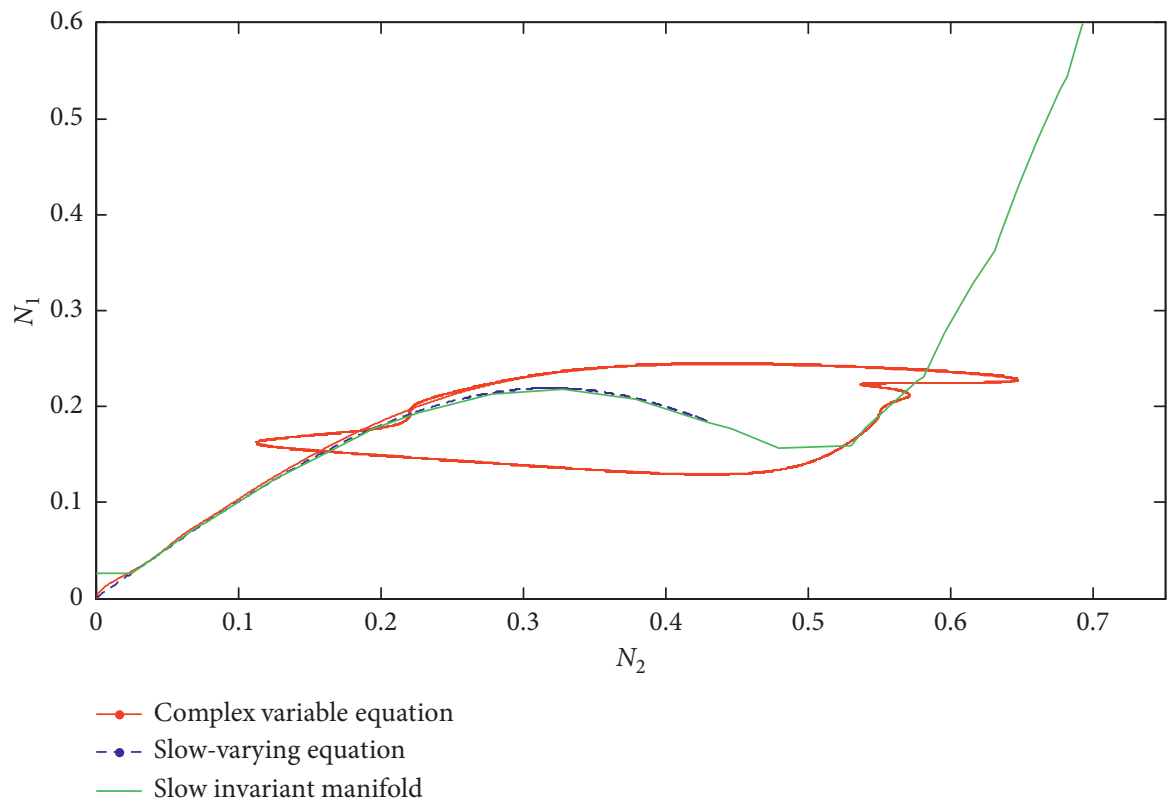

FIgURE 11: Slow invariant manifold of the system for the case $\lambda_{1}=0.8 ; \lambda_{2}=0.3 ; k n=5 ; f=0.5$.
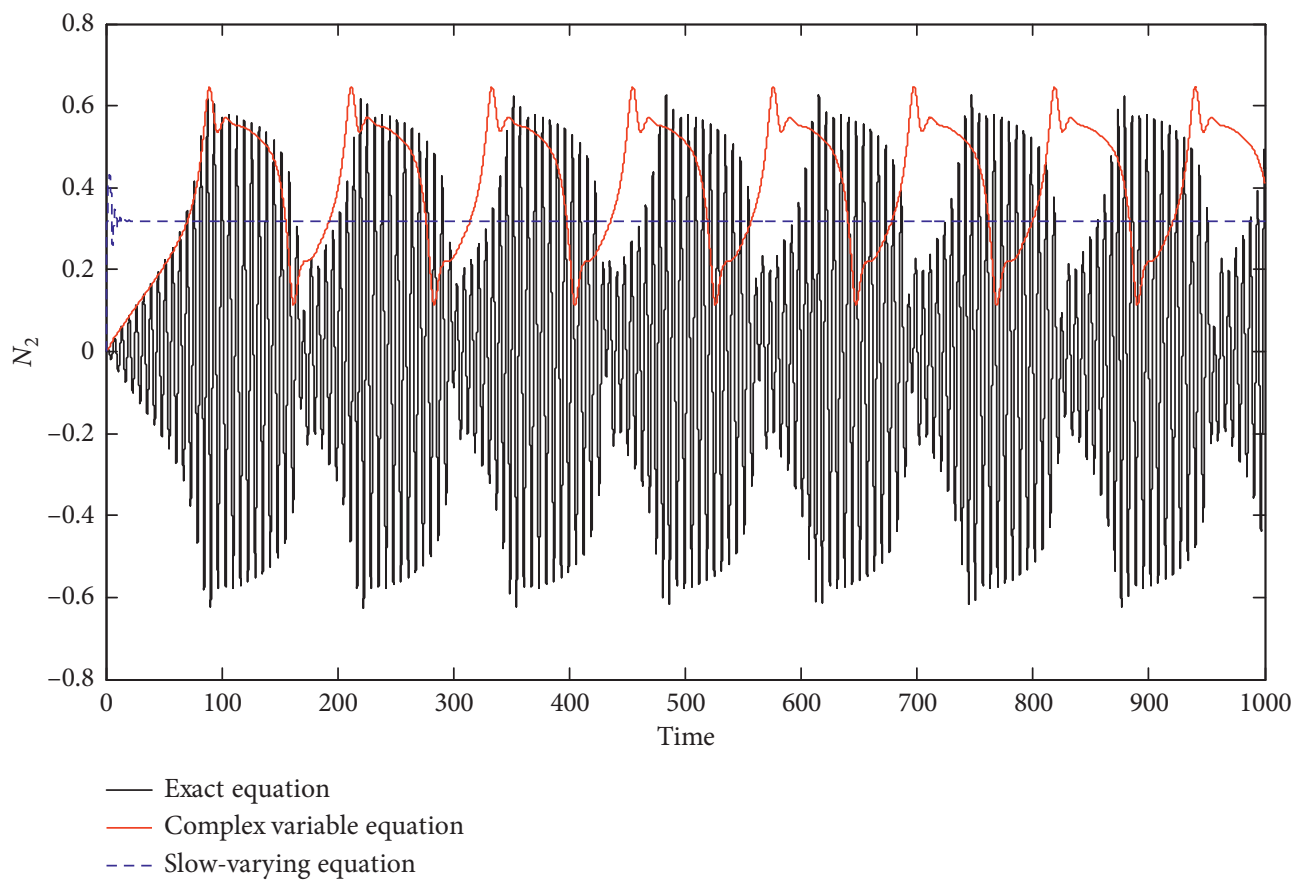

FIGURE 12: Time-history curves of nonlinear energy sink. The dashed line refers to the steady-state response of the slow-varying equation. System parameters: $\lambda_{1}=0.8 ; \lambda_{2}=0.3 ; k n=5 ; f=0.5$.

$$
\begin{aligned}
& f_{2}=f_{1}+\frac{C_{2}}{\cos \delta_{21}}, \\
& f_{2}=-f_{1}-\frac{D_{2}}{\sin \delta_{21}} .
\end{aligned}
$$

By using equations (42) and (43) at the starting and ending points of the platform, respectively, equation (58) can be obtained.

$$
\frac{C_{2}}{D_{2}}=\frac{\lambda_{2}}{(3 / 4) k n N_{21}^{2}-1} .
$$

Based on trigonometric identities, the following expression can be obtained.

$$
\left(C_{2}+F_{1}\right)^{2}+\left(D_{2}-F_{2}\right)^{2}=f_{2}^{2} .
$$

By substituting equations (56) and (58) into equation (59), the abscissa of the ending point $f_{2}$ can be found. 


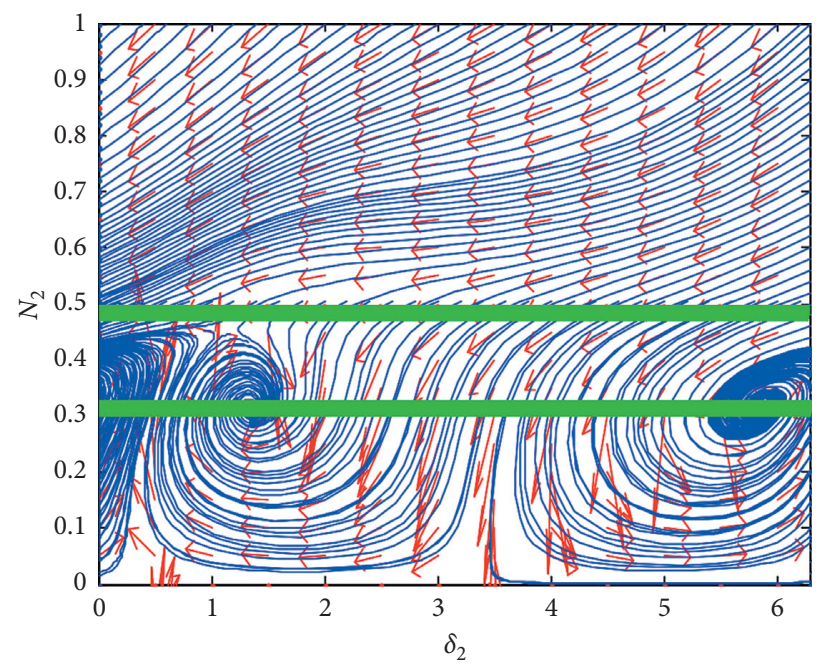

Figure 13: Phase portrait of nonlinear energy sink for the case $\lambda_{1}=0.8 ; \lambda_{2}=0.3 ; k n=5 ; f=0.5$.

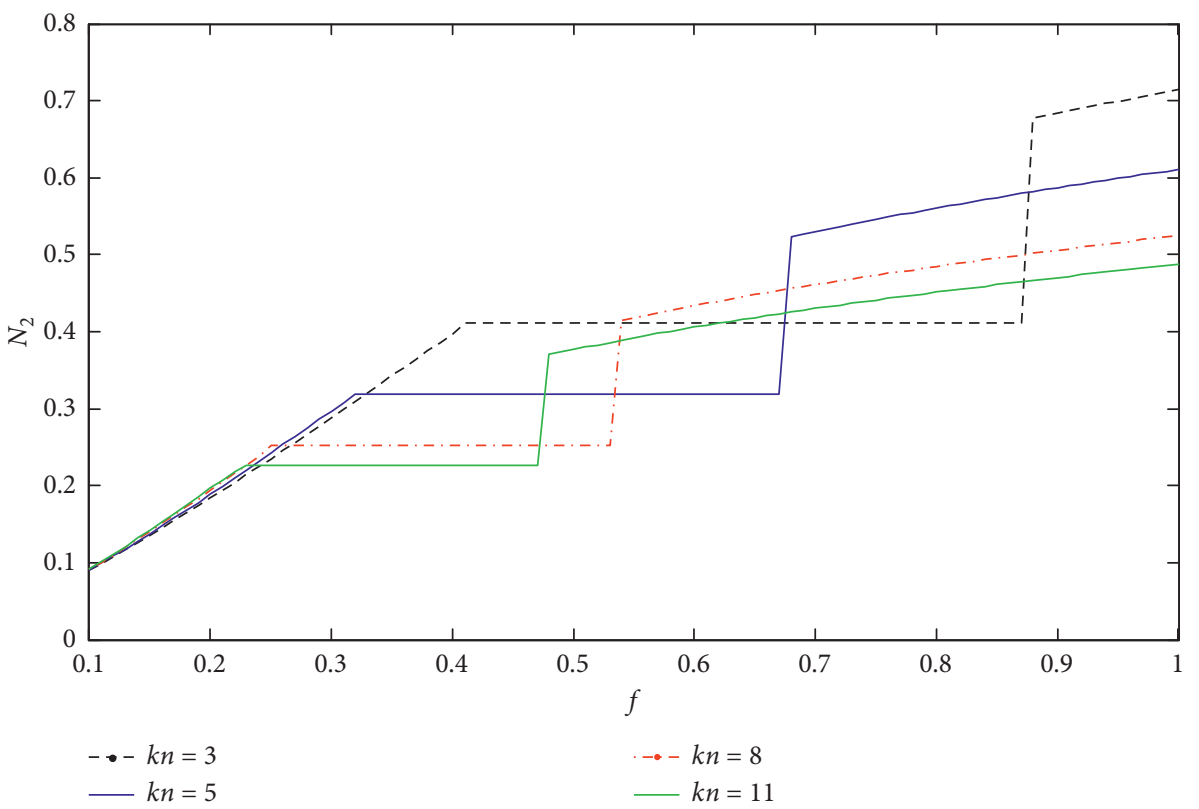

Figure 14: NES amplitude $N_{2}$ of different nonlinear stiffness $k n\left(\lambda_{1}=0.8, \lambda_{2}=0.3\right)$.

$f_{2}^{2}=\left[\left(f_{2}-f_{1}\right) \frac{F_{1}}{f_{1}}+F_{1}\right]^{2}+\left[\frac{\left(f_{2}-f_{1}\right)\left(3 / 4 k n N_{21}^{2}-1\right) F_{1}}{\lambda_{2} f_{1}}-F_{2}\right]^{2}$.

However, equation (60) can be reduced to equation (61).

$f_{2}^{2}=F_{1}^{2}\left\{\left(\frac{f_{2}}{f_{1}}\right)^{2}+\left[\frac{1}{\lambda_{2} f_{1}}\left(f_{2}-f_{1}\right)\left(\frac{3}{4} k n N_{21}^{2}-1\right)-\frac{F_{2}}{F_{1}}\right]^{2}\right\}$.

Therefore, the excitation amplitude region of the SMR is given as follows.

$$
f_{1} \leq f \leq f_{2} \text {. }
$$

Substituting $f_{1}$ and $f_{2}$ into the above inequality yields the following equation.

$$
\sqrt{F_{1}^{2}+F_{2}^{2}} \leq f \leq F_{1} \sqrt{\left(\frac{f_{2}}{f_{1}}\right)^{2}+\left[\frac{1}{\lambda_{2} f_{1}}\left(f_{2}-f_{1}\right)\left(\frac{3}{4} k n N_{21}^{2}-1\right)-\frac{F_{2}}{F_{1}}\right]^{2}} .
$$

Equation (63) is the equation for the excitation amplitude region of the SMR derived using the platform phenomenon. The accuracy of equation (63) is verified. 


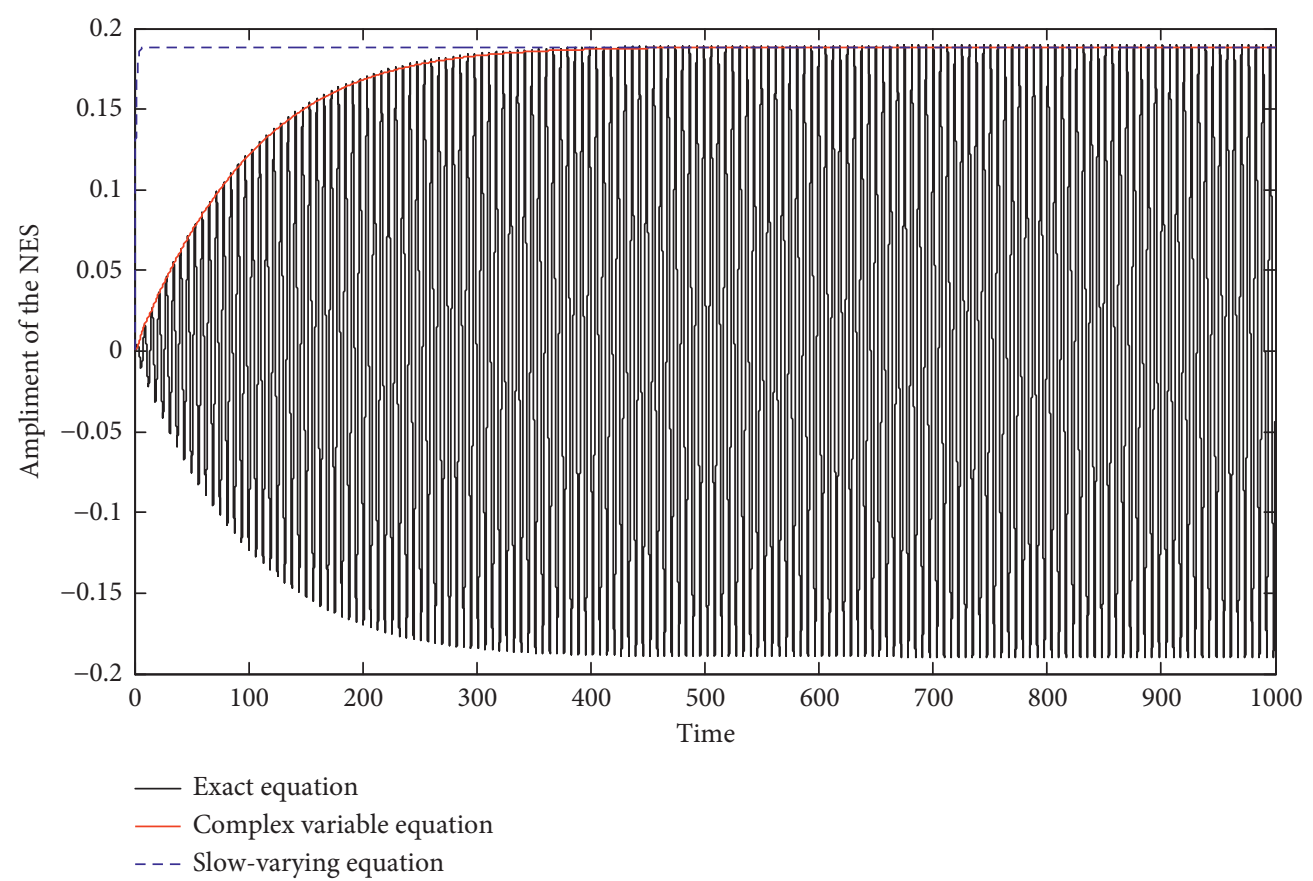

FIGURE 15: Time-history curves of nonlinear energy sink. The dashed line refers to the steady-state response of the slow-varying equation $(f=0.2)$.

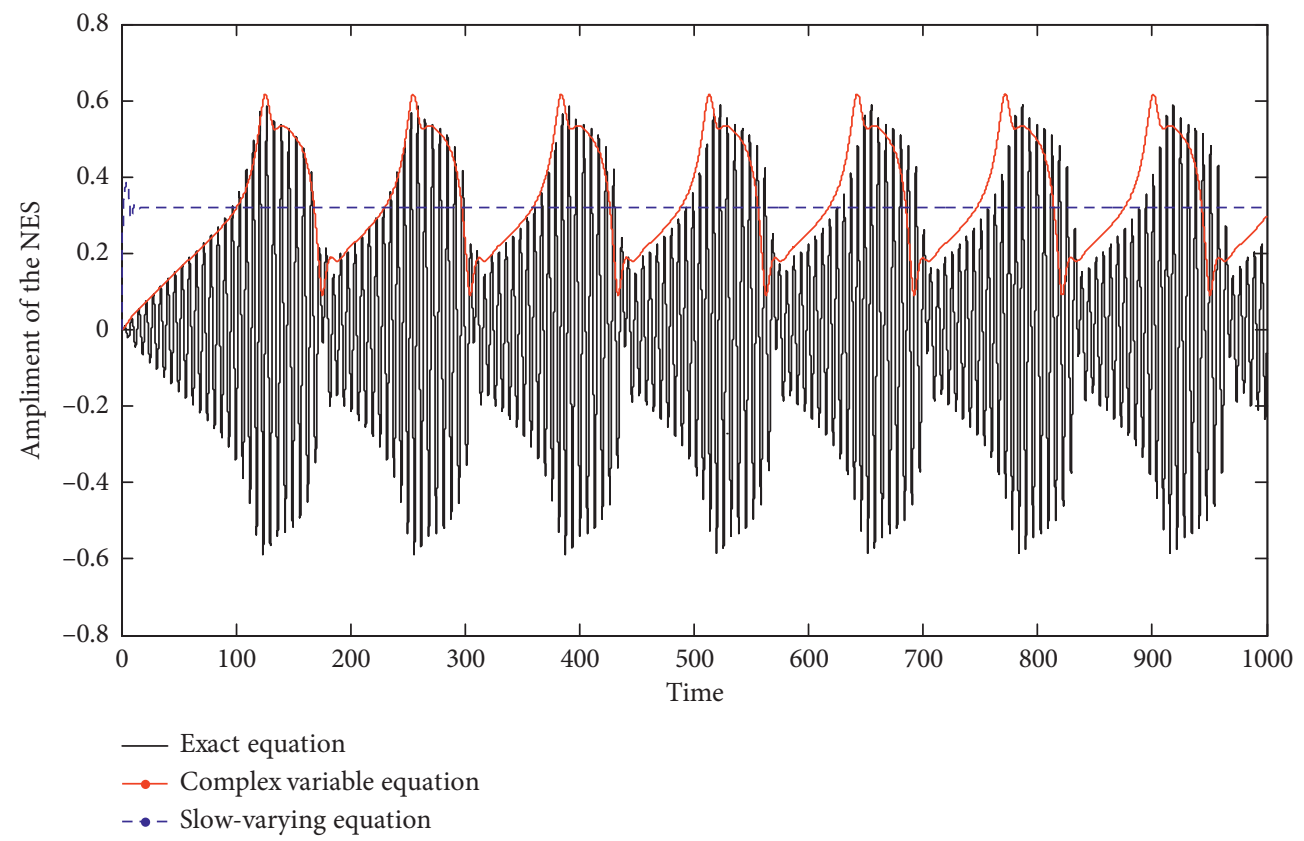

FiguRE 16: Time-history curves of nonlinear energy sink. The dashed line refers to the steady-state response of the slow-varying equation $(f=0.4)$.

The following tables show how $f_{1}$ and $f_{2}$ calculated using the numerical method and equation (63), respectively, when different nonlinear stiffness values are considered. Through calculations, the average errors of $f_{1}$ and $f_{2}$ are 0.0032 and 0.0322 , respectively. It can be noticed that the error of $f_{1}$ is significantly smaller than that of $f_{2}$. However, the error is within the acceptable range and have a minimal effect on the results of the subsequent optimizing stiffness $k n_{b}$. Furthermore, the larger the $k n$ value, the smaller the error. The values of the nonlinear stiffness $k n$ listed in Tables 1 and 2 and the $k n$ values shown in Figure 21 are the same.

Through the analysis in this section, the following conclusions can be drawn for a two-degree-freedom system which is composed of a linear primary structure and a cubic stiffness NES under harmonic excitation. 


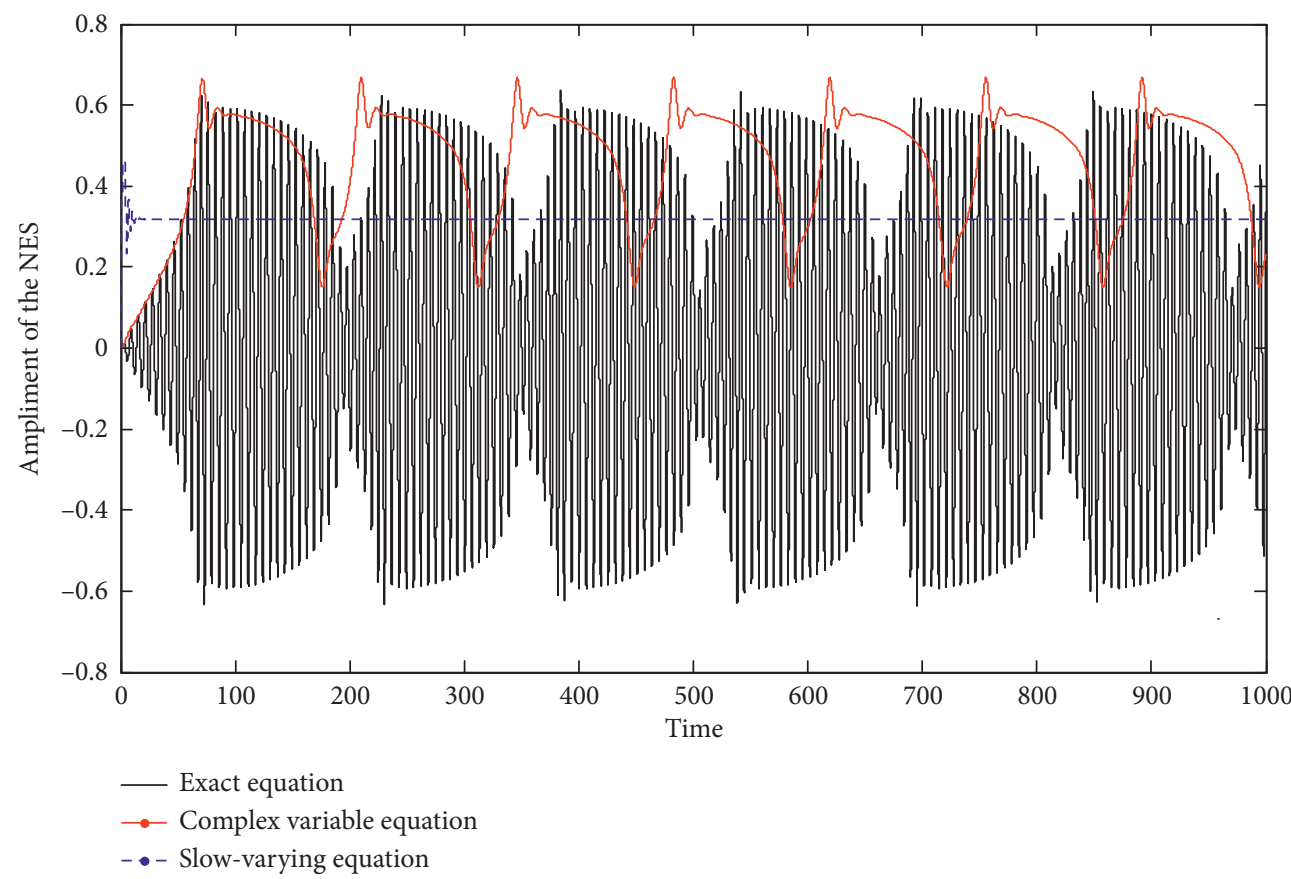

FIGURE 17: Time-history curves of nonlinear energy sink. The dashed line refers to the steady-state response of the slow-varying equation $(f=0.6)$.



FIgURE 18: Time-history curves of nonlinear energy sink. The dashed line refers to the steady-state response of the slow-varying equation $(f=0.8)$.

(1) When the system parameters are determined, there exists an interval $\left[f_{1}, f_{2}\right]$ of excitation amplitude $f$. The modulation response can occur in the steadystate phase, if and only if $f$ is in this interval. Moreover, the steady-state response is stable when $f$ is not in the interval. The curve " $f-N_{2}$ " is a horizontal straight line in the range of $\left[f_{1}, f_{2}\right]$, which is the so-called platform in this study. The range of the region is related to the nonlinear stiffness $k n$ of the NES.

(2) When the system parameters are determined, slowly varying phase $\delta_{2}$ shows a decreasing trend only in the 


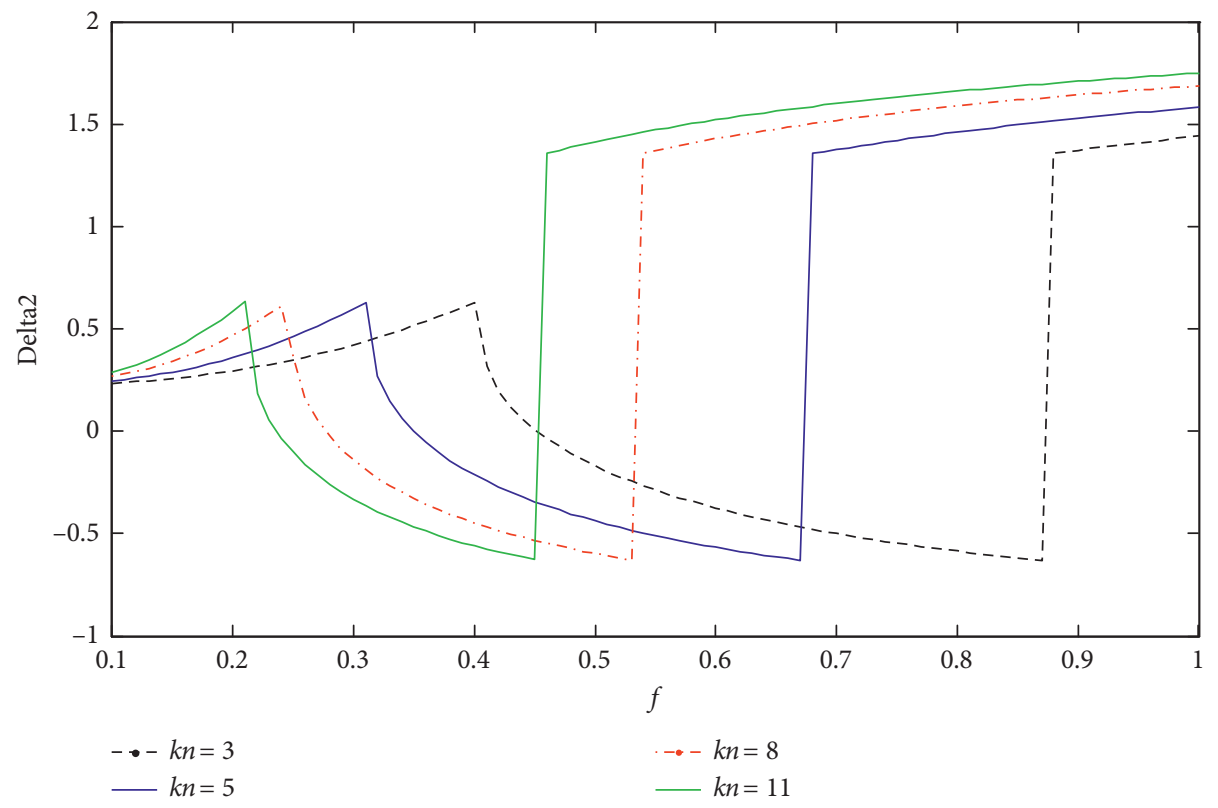

FIGURE 19: NES phase $\delta_{2}$ of different nonlinear stiffness $k n\left(\lambda_{1}=0.8, \lambda_{2}=0.3\right)$.

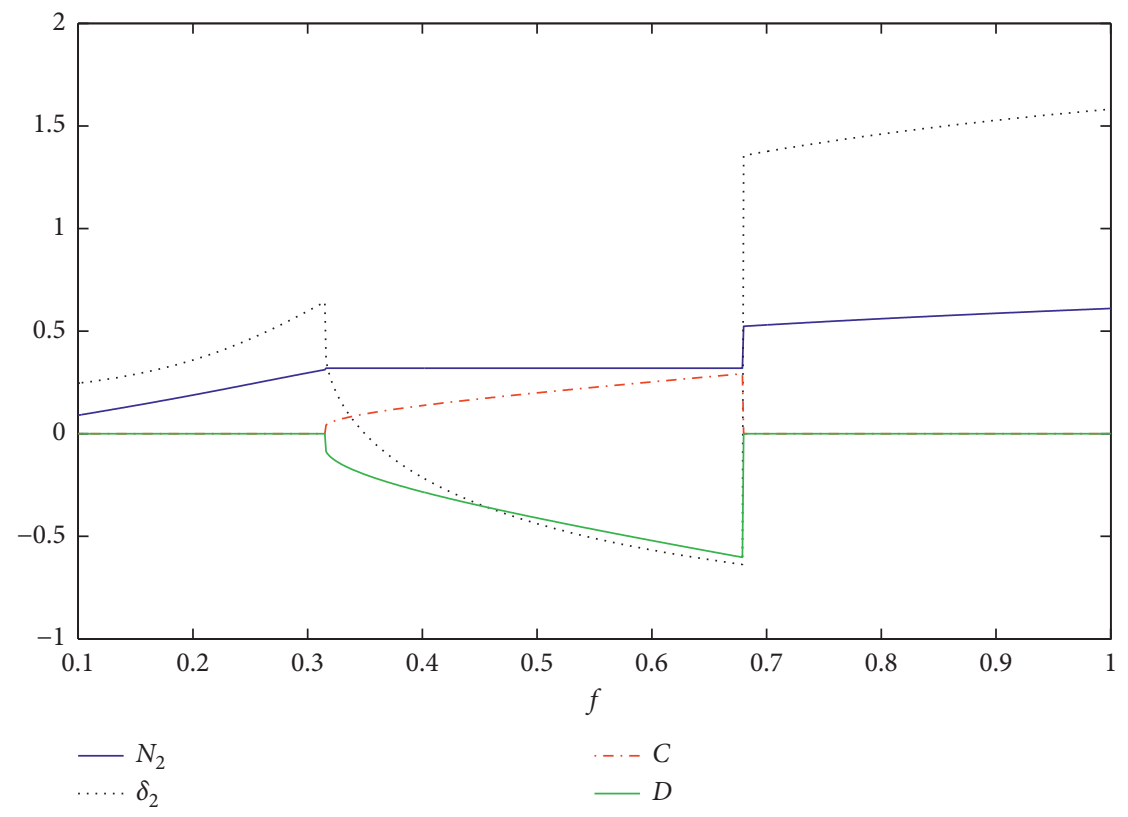

Figure 20: Comparison of NES amplitude $N_{2}$, NES phase $\delta_{2}$, nonlinear terms $C$ and $D$ for the case $\lambda_{1}=0.8, \lambda_{2}=0.3, k n=5$.

interval $\left[f_{1}, f_{2}\right]$. In this interval, the range of $\delta_{2}$ is independent of $k n$. The values of $\delta_{2}$ at the upper and lower bounds of $\left[f_{1}, f_{2}\right]$ are opposite to each other.

(3) The interval $\left[f_{1}, f_{2}\right]$ can be obtained from the above conclusions, which is the excitation amplitude range of the modulation response of the system

\section{Optimal Stiffness of NES}

As shown in Figures 14 and 21, the values of $f_{1}, f_{2}$ are related to $k n$. The smaller the $k n$ value, the broader the platform. It is a good phenomenon if the platform becomes wider. However, the steady-state amplitude of the primary structure increases simultaneously. A trade-off relationship exists between the platform width and steady-state amplitude. Thus, it can be inferred that the nonlinear stiffness is not better when it becomes larger or smaller. A range of $k n$ must exist within which the valid TET will occur.

Because $f_{1}$ and $f_{2}$ are analytical expressions of $\lambda_{1}, \lambda_{2}$, and $k n$, a range of effective stiffness can be obtained using equation (63) when $\lambda 1$ and $\lambda_{2}$ are determined, and the effective stiffness range of the response can be set as $\left[k n_{1}, k n_{2}\right]$. As can be observed from Figure 14, when the excitation amplitude $f$ is determined, finding the platform with $f$ as 
TABLE 1: Error of $f_{1}$.

\begin{tabular}{lcccccc}
\hline$k n$ & 3 & 5 & 7 & 9 & 11 & 13 \\
\hline Analytic solutions & 0.4157 & 0.3220 & 0.2721 & 0.2400 & 0.2171 & 0.1997 \\
Numerical solutions & 0.4121 & 0.3181 & 0.2701 & 0.2360 & 0.2140 & 0.1970 \\
\hline
\end{tabular}

TABLE 2: Error of $f_{2}$.

\begin{tabular}{lcccccc}
\hline$k n$ & 3 & 5 & 7 & 9 & 11 & 13 \\
\hline Analytic solutions & 0.9249 & 0.7164 & 0.6055 & 0.5340 & 0.4830 & 0.4443 \\
Numerical solutions & 0.8770 & 0.6790 & 0.5730 & 0.5050 & 0.4570 & 0.4200 \\
\hline
\end{tabular}

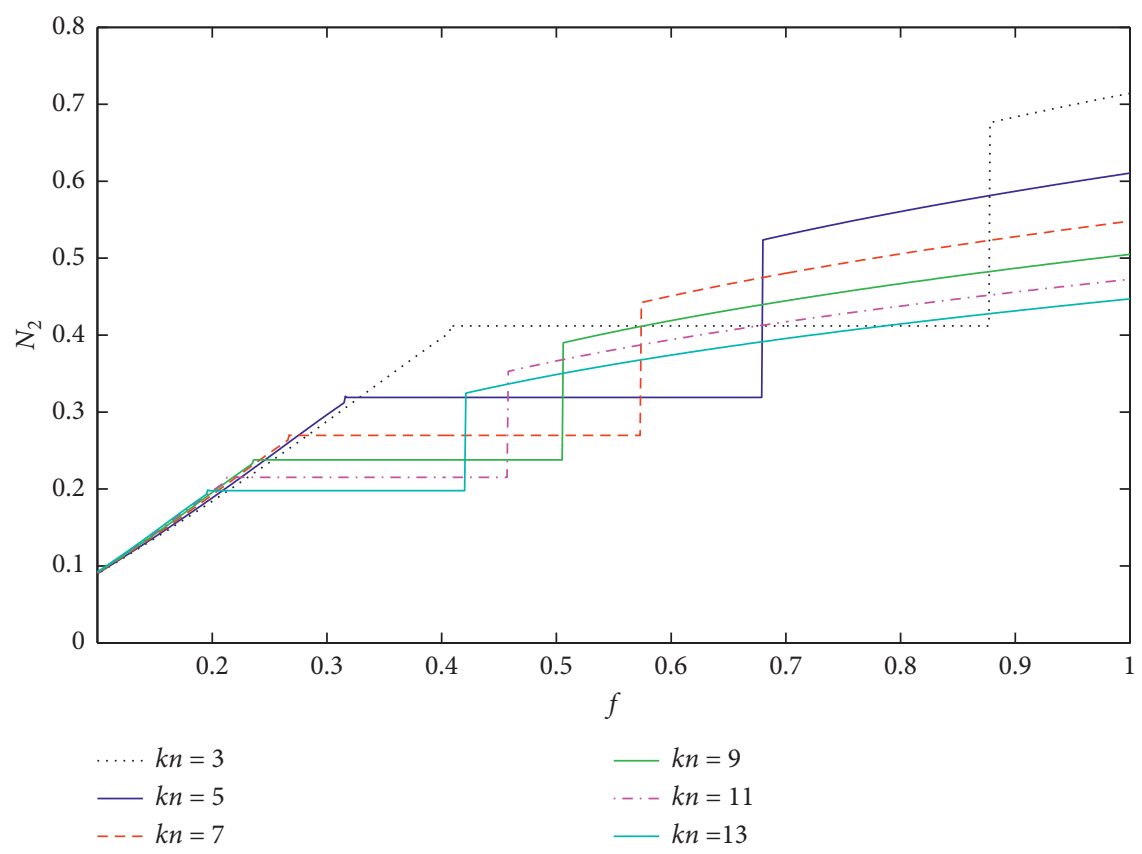

Figure 21: NES amplitude $N_{2}$ of different nonlinear stiffness $k n\left(\lambda_{1}=0.8, \lambda_{2}=0.3\right)$.

the abscissa of the endpoint and the corresponding stiffness of this platform is regarded as the optimal stiffness appears to be reasonable. However, as the platform range obtained using equation (63) is only an approximate solution, it can be observed from Tables 1 and 2 that some inescapable errors occur, especially for $f_{2}$. Thus, a significant error must occur to consider $k n_{2}$ as the optimal stiffness. Therefore, in this study, the average value of the sum of $k n_{1}$ and $k n_{2}$ is considered as the optimization stiffness $k n_{b}$, which not only ensures that the steady-state response of the system is SMR but also ensures that the system can cope with the fluctuation of the excitation amplitude within a small range and improve robustness. Several amplitudes of $f$ are considered, and equation (63) is used to obtain $k n_{1}, k n_{2}$, and $k n_{b}$ under these working conditions. The results are listed in Table 3.

\section{Numerical Analysis of Stiffness Optimization Results}

The optimization effect of $k n_{b}$ was verified using the numerical method by selecting the excitation amplitude as $f=0.5$ and considering four values of $k n_{b}$, i.e.,
TABLE 3: Optimal nonlinear stiffness $k n_{b}, f_{2}$.

\begin{tabular}{lccccccc}
\hline$f$ & 0.3 & 0.4 & 0.5 & 0.6 & 0.7 & 0.8 & 0.9 \\
\hline$k n_{1}$ & 5.7589 & 3.2394 & 2.0732 & 1.4397 & 1.0578 & 0.8098 & 0.6399 \\
$k n_{2}$ & 28.5138 & 16.0390 & 10.2650 & 7.1285 & 5.2372 & 4.0098 & 3.1682 \\
$k n_{b}$ & 17.1363 & 9.6392 & 6.1691 & 4.2841 & 3.1475 & 2.4098 & 1.9040 \\
\hline
\end{tabular}

$k n=1<k n_{1}, k n_{1}<k n_{b}=6.1691<k n_{2}, k n=k n_{2}=10.2650$, and $k n=14,18>k n_{2}$. As no fixed amplitude response of the SMR occurs, the average value of the total energy is used as a measure of the vibration reduction effect. The transient total energy of the system can be expressed as

$$
E(t)=\frac{1}{2} \dot{u}^{2}+\frac{1}{2}\left(\frac{u+\varepsilon v}{1+\varepsilon}\right)^{2}+\frac{\varepsilon^{2} k_{n}}{4} v^{4}
$$

Figure 22 shows the result obtained by changing the tuning parameters $\sigma$ and testing the average total energy near the $1: 1$ internal resonance.

It can be observed from Figure 19 that the total energy is significantly lower when the optimal stiffness value is considered. The optimized frequency-energy curve is plotted separately in Figure 23. The smooth areas on both sides are the stable steady-state responses, and the location near the 


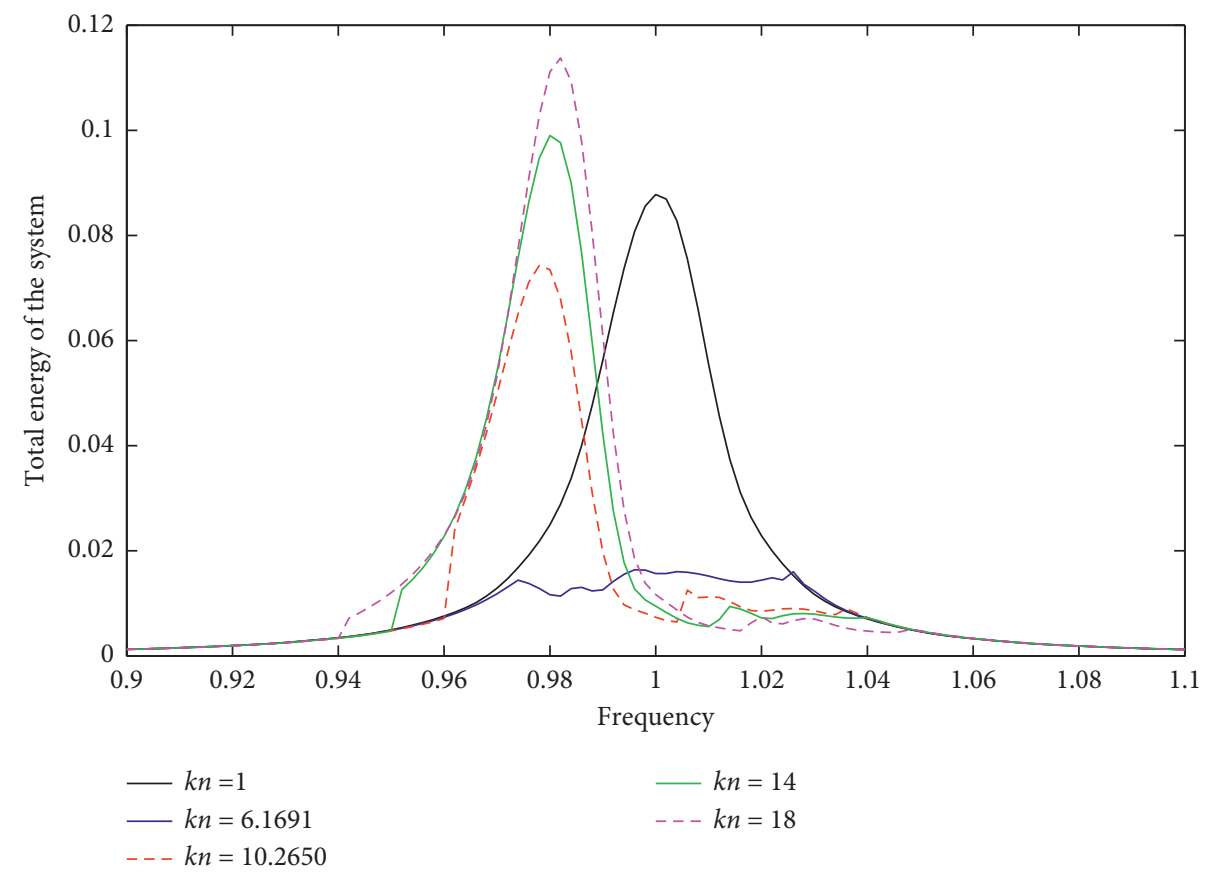

Figure 22: Average total energy of the system with respect to excitation frequency.

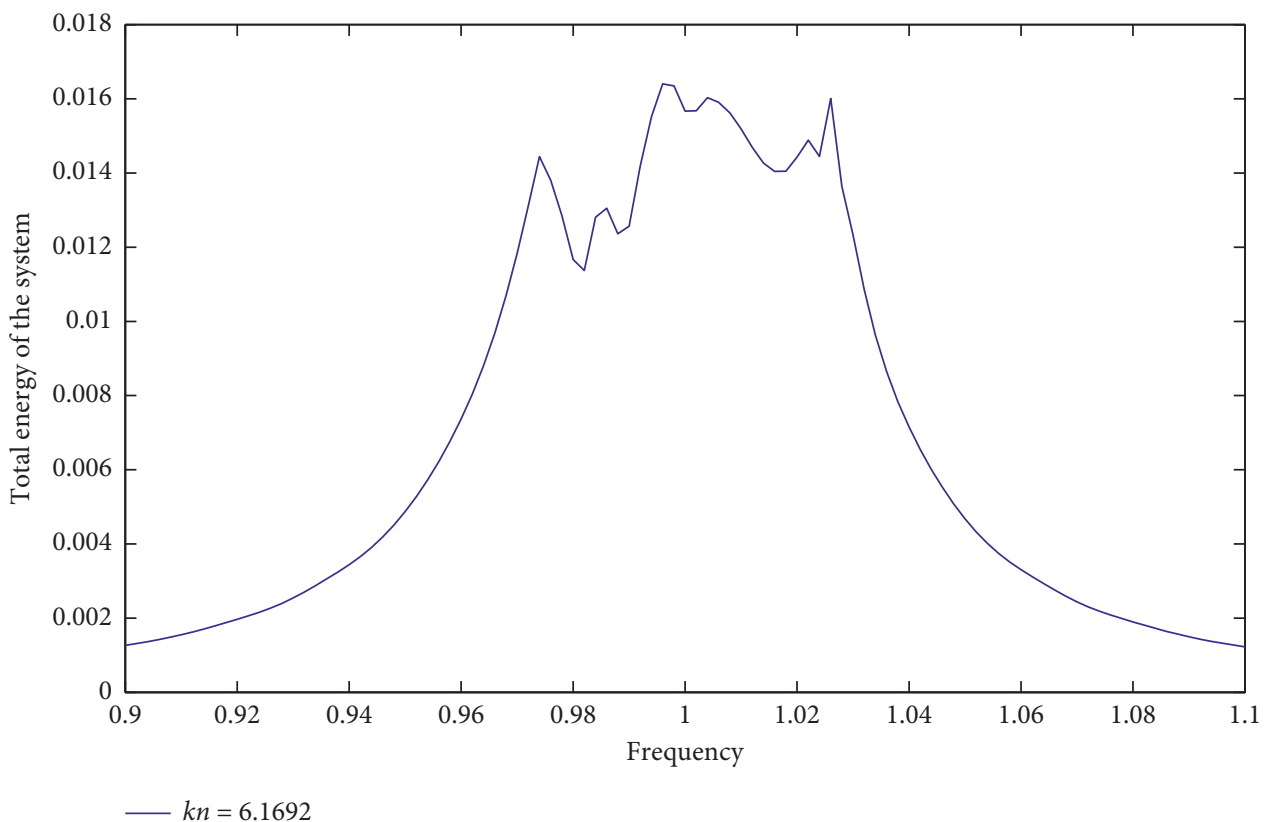

FIGURE 23: Average total energy of the system with respect to excitation frequency $\left(k n=k n_{b}\right)$.

middle represents the SMR; this response mode is beneficial for reducing vibration. In the case of $\omega=1$, the total energy of $k n_{b}$ is relatively small, but it is not the minimum value. Under actual conditions, the excitation frequency is probably not exactly equal to 1 . Thus, the robustness in the frequency domain is critical. Selecting $k n=k n_{b}$ achieves a balance between the effects of the vibration reduction and robustness on frequency.

By considering an excitation amplitude of $f=0.8$ and selecting the corresponding five values of $k n$ to verify the vibration reduction effect of $k n_{b}$, the conclusion inferred from Figures 24 and 25 is consistent with Figures 22 and 23. 


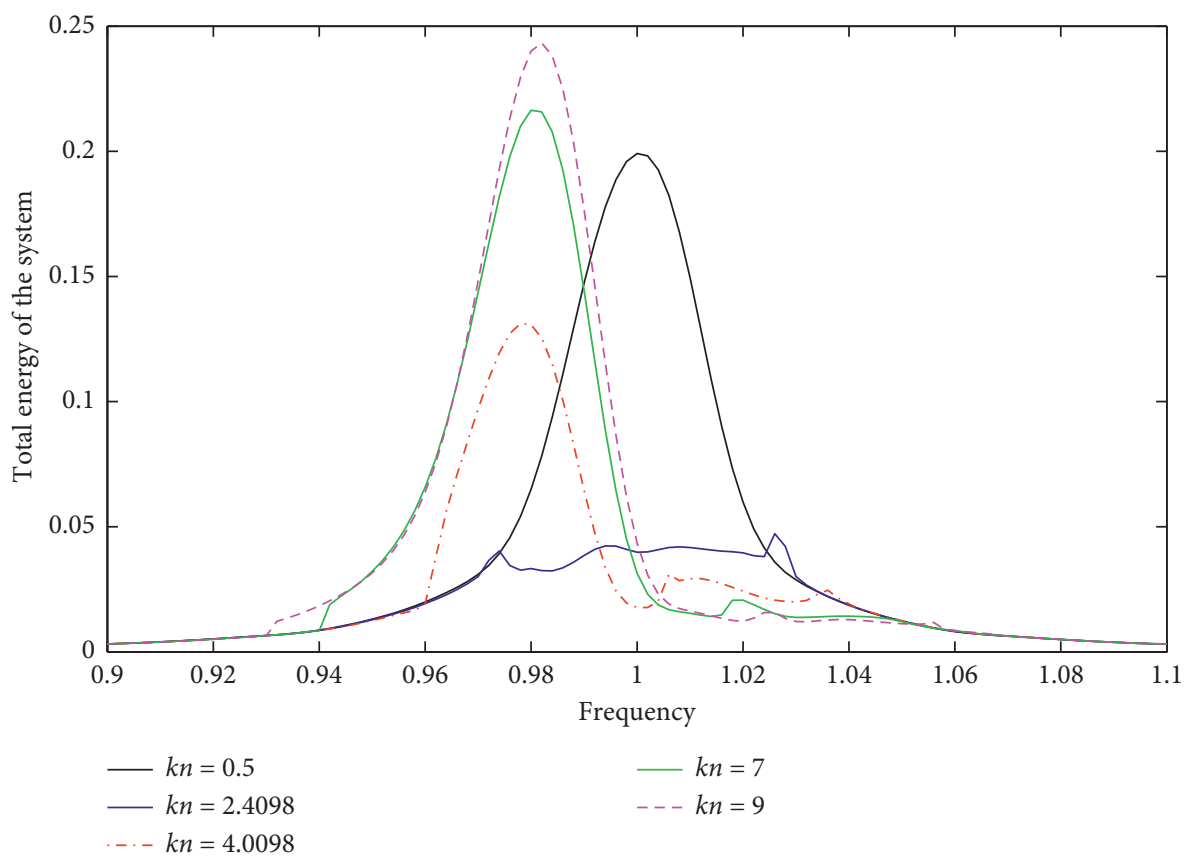

Figure 24: Average total energy of the system with respect to excitation frequency.

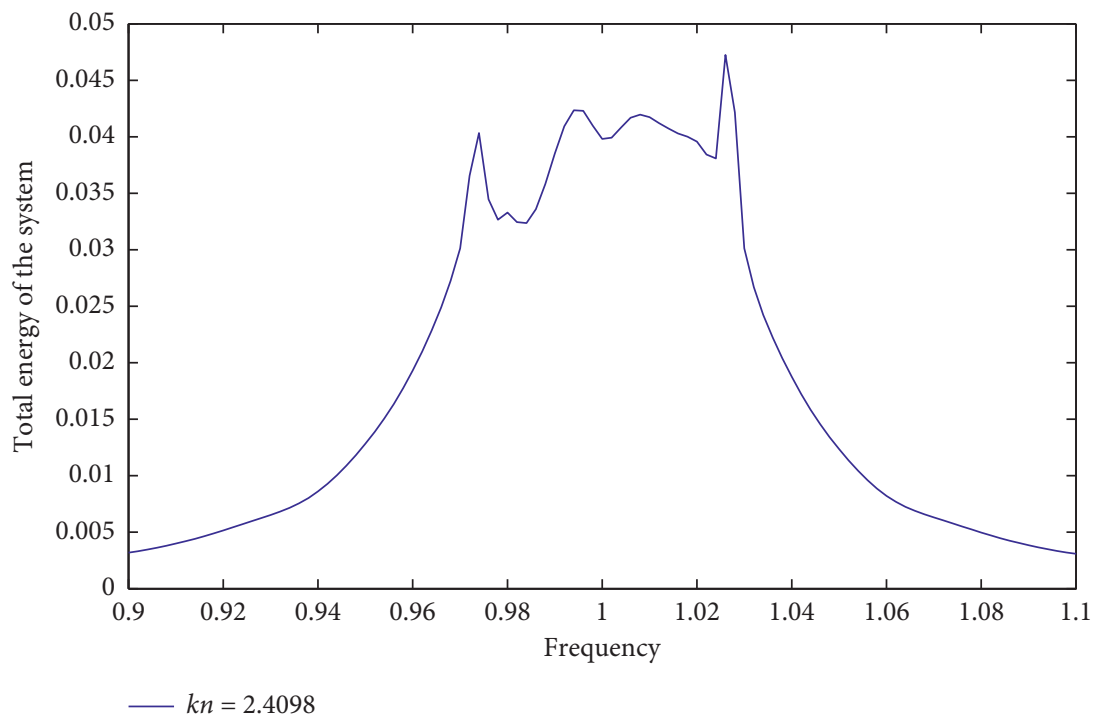

Figure 25: Average total energy of the system with respect to excitation frequency $\left(k n=k n_{b}\right)$.

\section{Conclusion}

This study presents an analysis of a 2-DOF system under harmonic excitation composed of a linear primary structure and a NES using the CX-A and multiscale method. The initial conditions of this system are always approximately zero, and the fast variables that are not useful for analytical analysis are eliminated. While studying the variation trends of the NES amplitude $N_{2}$ calculated using the slowly varying equation of the system with the excitation amplitude $f$, a platform phenomenon and other related phenomena are determined. In the platform, the steady-state amplitude $N_{2}$ obtained using the slow-varying equations is inconsistent with the actual response and do not change with $f$. Moreover, it is always fixed at the SN bifurcation point, and the phase $\delta_{2}$ at the starting and ending points of the platform can be regarded as negative. Based on the platform phenomenon, the region $\left[f_{1}, f_{2}\right]$ of the excitation amplitude in which the SMR can occur is obtained, and the accuracy of this range is verified numerically. 
About the platform phenomenon, the following conclusions can be drawn:

(1) When the system parameters are determined, there exists an interval $\left[f_{1}, f_{2}\right]$ of excitation amplitude $f$, and the modulation response can occur in the steady-state phase if and only if $f$ is in this interval. The range of the region is related to the nonlinear stiffness $k n$ of the NES.

(2) The slowly varying phase $\delta_{2}$ shows a decreasing trend only in the range of $\left[f_{1}, f_{2}\right]$, and the value of $\delta_{2}$ at the upper and lower bounds of $\left[f_{1}, f_{2}\right]$ are opposite to each other. The range of $\delta_{2}$ on $\left[f_{1}, f_{2}\right]$ is independent of $k n$.

(3) The range $\left[f_{1}, f_{2}\right]$ can be obtained from the above conclusions, which is the excitation amplitude range of the modulation response of the system.

After the excitation range of SMR is obtained, the approximate analytical solution of the optimal nonlinear stiffness $k n_{b}$ can be determined when other parameters are fixed. Finally, the average value of the total energy is adopted as the measurement, and the superior vibration reduction effect of the optimized stiffness $k n_{b}$ is verified using the numerical method.

It is the first time that the platform phenomenon is put forward. Some laws related to this phenomenon have great research and application value and play a crucial role in the parameter design of the NES. More laws about this phenomenon and the deeper physical meaning leading to this phenomenon still need to be explored.

\section{Data Availability}

The data, models, or codes that support the findings of this study are available from the corresponding author upon request.

\section{Conflicts of Interest}

The authors declare that they have no conflicts of interest.

\section{Acknowledgments}

This study was supported in part by the National Natural Science Foundation of China (51678116).

\section{References}

[1] K. C. S. Kwok and B. Samali, "Performance of tuned mass dampers under wind loads," Engineering Structures, vol. 17, no. 9, pp. 655-667, 1995.

[2] Q. S. Li, L.-H. Zhi, A. Y. Tuan, and C. S. Kao, "Dynamic behavior of Taipei 101 tower: field measurement and numerical analysis," Journal of Structural Engineering, ASCE, vol. 137, no. 1, pp. 143-155, 2011.

[3] J. P. Den Hartog, Mechanical Vibrations, McGraw-Hill, New York, NY, USA, 1947.

[4] H. C. Tsai and G. C. Lin, "Optimum tuned mass damper for minimizing steady-state response of support-excited and damped systems," Earthquake Engineering \& Structural Dynamics, vol. 22, pp. 957-973, 1993.

[5] S. Fallahpasand, M. Dardel, M. H. Pashaei, and H. Danali, "Investigation and optimization of nonlinear pendulum vibration absorber for horizontal vibration suppression of damped system," The Structural Design of Tall and Special Buildings, vol. 24, no. 14, pp. 873-893, 2015.

[6] M. Iizuka, "Simple design formulas for mechanical properties of laminated low-damping rubber bearing used in base isolation," Technical Report, pp. 521-522, Summaries of Technical Papers of Annual Meeting of the Architectural Institute of Japan, Tokyo, Japan, 1993.

[7] J. Teng, Z. X. Lu, Y. Q. Xiao et al., "Vibration control of highrising buildings with TMD considering contact nonlinear damping effect," Vibration and Shock, vol. 28, no. 3, pp. 90-97, 2009.

[8] L. Li and P. Cui, "Novel design approach of a nonlinear tuned mass damper with duffing stiffness," Journal of Engineering Mechanics, vol. 143, no. 4, Article ID 04017004, 2017.

[9] L. Y. Li and T. Zhang, "Analytical analysis for the design of nonlinear tuned mass damper," Journal of Vibration and Control, vol. 26, no. 10, Article ID 107754631988984, 2020.

[10] R. Roberson, "Synthesis of a nonlinear dynamic vibration absorber," Journal of the Franklin Institute, vol. 254, no. 5, pp. 205-220, 1952.

[11] P. Lieber and D. P. Jensen, Transactions ASME, vol. 67, p. 523, 1945.

[12] R. D. Nayeri, S. F. Masri, and J. P. Caffrey, "Studies of the performance of multi-unit impact dampers under stochastic excitation," Journal of Vibration Acoustics, vol. 129, no. 2, pp. 239-251, 2007.

[13] A. Papalou and S. F. Masri, "Performance of particle dampers under random excitation," Journal of Vibration Acoustics ASME, vol. 118, no. 4, pp. 614-621, 1996.

[14] S. F. Masri, "Periodic excitation of multiple-unit impact dampers," American Society of Civil Engineers, vol. 96, pp. 1195-1207, 1970.

[15] L. Zheng, W. Zixin, F. M. Sami, and L. Xilin, "Particle impact dampers: past, present, and future," Structural Control and Health Monitoring, vol. 25, 2017.

[16] O. V. Gendelman, Y. Starosvetsky, and M. Feldman, "Attractors of harmonically forced linear oscillator with attached nonlinear energy sink I: description of response regimes," Nonlinear Dynamics, vol. 51, no. 1-2, pp. 31-46, 2008.

[17] Y. Starosvetsky and O. V. Gendelman, "Attractors of harmonically forced linear oscillator with attached nonlinear energy sink. II: optimization of a nonlinear vibration absorber," Nonlinear Dynamics, vol. 51, no. 1-2, pp. 47-57, 2008.

[18] Y. Starosvetsky and O. V. Gendelman, "Strongly modulated response in forced $2 \mathrm{DOF}$ oscillatory system with essential mass and potential asymmetry," Physical D Nonlinear Phenomena, vol. 237, no. 13, pp. 1719-1733, 2008.

[19] Y. Starosvetsky and O. V. Gendelman, "Response regimes of linear oscillator coupled to nonlinear energy sink with harmonic forcing and frequency detuning," Journal of Sound and Vibration, vol. 315, no. 3, pp. 746-765, 2008.

[20] L. K. Liu, P. Tan, Y. Y. Chen, W. Yan, and F. Zhou, "Strongly modulated response analysis of nonlinear energy sink absorption system under ground harmonic excitation," Journal of Beijing University of Technology, vol. 45, no. 2, pp. 177-185, 2019.

[21] Y. C. Zhang, X. R. Kong, Z. X. Yang, and H. L. Zhang, "Targeted energy transfer and parameter design of a nonlinear 
vibration absorber," Journal of Vibration Engineering, vol. 2, pp. 111-117, 2011.

[22] Y. C. Zhang, X. R. Kong, and H. L. Zhang, "Targeted energy transfer among coupled nonlinear oscillators complete energy exchange in a conservative system," Journal of Vibration and Shock, vol. 31, no. 1, pp. 150-155, 2012.

[23] Y. C. Zhang, Research on the dynamics and performance of vibration suppresion of nonliner energy sink, $\mathrm{PhD}$ thesis, Harbin Institute of Technology, Harbin, China, 2012.

[24] Y. C. Zhang, "Dynamics of a nonlinear energy sink used for suppressing two-separated resonance peaks," Spacecraft Environment Engineering, vol. 32, no. 5, pp. 477-483, 2015.

[25] Y. C. Zhang and X. R. Kong, "Initial conditions for targeted energy transfer in coupled nonlinear oscillators," Journal of Harbin Institute of Technology, vol. 44, no. 7, pp. 21-26, 2012.

[26] Y. C. Zhang and X. R. Kong, "Vibration suppression of a twodegree-of-freedom nonlinear energy sink under harmonic excitation," Acta Aeronautica et Astronautica Sinica, vol. 33, no. 6, pp. 1020-1029, 2012.

[27] A. Shudeifat and A. Mohammad, "Highly efficient nonlinear energy sink," Nonlinear Dynamics, vol. 76 , no. 4, pp. 1905-1920, 2014.

[28] W. G. Molyneaux, Supports for Vibration Isolation, AER Research Council, London, UK, 1957.

[29] D. L. Platus, "Negative-stiffness-mechanism vibration isolation systems," Proceedings of SPIE-The International Society for Optical Engineering, vol. 3786, pp. 44-54, 1999.

[30] L. I. Manevitch, A. I. Musienko, and C. Lamarque, "New analytical approach to energy pumping problem in strongly nonhomogeneous 2 dof systems," Meccanica, vol. 42, no. 1, pp. 77-83, 2007.

[31] Y. Niu, Y. Hao, M. Yao, W. Zhang, and S. Yang, "Nonlinear dynamics of imperfect fgm conical panel," Shock and Vibration, vol. 2018, no. 2, Article ID 4187386, 20 pages, 2018.

[32] Z. Q. Lu, H. Ding, and L. Q. Chen, "Resonance response interaction without internal resonance in vibratory energy harvesting," Mechanical Systems and Signal Processing, vol. 121, no. 15, pp. 767-776, 2019.

[33] D. D. Tan, Z. Q. Lu, D. H. Gu, H. Ding, and L. Q. Chen, "A ring vibration isolator enhanced by a nonlinear energy sink," Journal of Sound and Vibration, vol. 508, no. 1-3, Article ID 116201, 2021.

[34] D. Qiu, S. Seguy, and M. Paredes, "Design criteria for optimally tuned vibro-impact nonlinear energy sink," Journal of Sound and Vibration, vol. 442, pp. 497-513, 2018.

[35] D. Qiu, M. Paredes, and S. Seguy, "Variable pitch spring for nonlinear energy sink: application to passive vibration control," Archive Proceedings of the Institution of Mechanical Engineers Part C Journal of Mechanical Engineering Science 1989-1996, vol. 203-210, Article ID 095440621876148, 2018. 